\title{
Ionospheric energy input as a function of solar wind parameters: global MHD simulation results
}

\author{
M. Palmroth ${ }^{1}$, P. Janhunen ${ }^{1}$, T. I. Pulkkinen ${ }^{1}$, and H. E. J. Koskinen ${ }^{2,1}$ \\ ${ }^{1}$ Finnish Meteorological Institute, Geophysical Research Division, Finland \\ ${ }^{2}$ University of Helsinki, Department of Physical Sciences, Finland
}

Received: 17 December 2002 - Revised: 29 April 2003 - Accepted: 18 June 2003 - Published: 1 January 2004

\begin{abstract}
We examine the global energetics of the solar wind magnetosphere-ionosphere system by using the global MHD simulation code GUMICS-4. We show simulation results for a major magnetospheric storm (6 April 2000) and a moderate substorm (15 August 2001). The ionospheric dissipation is investigated by determining the Joule heating and precipitation powers in the simulation during the two events. The ionospheric dissipation is concentrated largely on the dayside cusp region during the main phase of the storm period, whereas the nightside oval dominates the ionospheric dissipation during the substorm event. The temporal variations of the precipitation power during the two events are shown to correlate well with the commonly used $A E$-based proxy of the precipitation power. The temporal variation of the Joule heating power during the substorm event is wellcorrelated with a commonly used $A E$-based empirical proxy, whereas during the storm period the simulated Joule heating is different from the empirical proxy. Finally, we derive a power law formula, which gives the total ionospheric dissipation from the solar wind density, velocity and magnetic field $z$-component and which agrees with the simulation result with more than $80 \%$ correlation.
\end{abstract}

Key words. Ionosphere (modeling and forecasting) Magnetospheric physics (magnetosphere-ionosphere interactions; storms and substorms)

\section{Introduction}

The energy transfer process between the solar wind and the magnetosphere and further between the ionosphere is one of the key questions in space physics, frequently brought up in executive summaries of many proposals and space physics research strategy reports (e.g. Acuña et al., 1995). While the energy transfer process was qualitatively explained already in the 1960's by the first theories of the solar windmagnetosphere coupling (Dungey, 1961; Axford and Hines,

Correspondence to: M. Palmroth (Minna.Palmroth@fmi.fi
1961), the quantitative assessment of the problem has proven to be difficult. The energy transfer mechanism by which the solar wind energy enters the magnetosphere has been explained by magnetic reconnection and viscous interaction. The amount of transferred energy is still uncertain, because the results rely on correlations of solar wind parameters with known dissipation channels inside the magnetosphere (e.g. Akasofu, 1981). The first quantitative attempt using a global MHD simulation to identify both the amount of energy transferred through the magnetopause as well as the energy transfer locations at the magnetopause was made by Palmroth et al. (2003). They found that during southward interplanetary magnetic field (IMF) the locations of energy transfer are controlled by the focusing of the Poynting vector in the plane of the IMF clock angle (see also Papadopoulos et al., 1999). On the other hand, during northward IMF the Poynting flux focusing does not play a major role in determining the energy transfer locations, as reconnection may not have opened the magnetopause at the locations where the Poynting vector focuses (Palmroth et al., 2003).

The dissipation of the solar wind energy, both during magnetospheric substorms and magnetic storms, in the various sinks in the magnetosphere and the ionosphere, has also been a subject of several past studies (Akasofu, 1981; Weiss et al., 1992; Lu et al., 1998; Turner et al., 2001; Pulkkinen et al., 2002). The understanding of the relative importance of the various sinks has changed over the years. For a long time the ring current was assumed to be the largest sink (Akasofu, 1981), whereas the more recent studies suggest that the polar ionosphere plays a major role in dissipating the solar wind energy (e.g. Weiss et al, 1992 and references therein). In the ionosphere the two largest dissipation mechanisms are the Ohmic Joule heating in the ionosphere, when the fieldaligned currents are closed across the equipotential surfaces, and the energy deposition by particles precipitating in the auroral region of the ionosphere.

The current understanding is that Joule heating consumes, on average, more energy than particle precipitation (e.g. Lu et al., 1998), but the estimates for the relative importance of 
the particle precipitation have substantially increased from the $1 \%$ assumed originally (e.g. Akasofu, 1981). At present, there are no direct ways to measure the energy deposited by Joule heating, only statistical estimates exist (e.g. Ahn et al., 1983), which give the amount of energy based on the ground magnetic variations caused by the auroral electrojets (such as the $A E$ index). The $A E$ index-based methods are only as good as the ability of the $A E$ index to describe the temporal and spatial variations of the Pedersen currents not only within the auroral regions, but in the polar cap as well. As the $A E$ stations are located at high latitudes, the true intensity of the auroral electrojets is not recorded, particularly during major storms when the auroral oval moves significantly equatorward.

Measuring the energy deposited by particle precipitation is easier than measuring the energy deposited by Joule heating. When precipitating into the ionosphere, particles collide with atmospheric particles which emit auroral light that can be directly measured on the ground or from polar-orbiting satellites. A method based on ultraviolet image measurements on board the Polar satellite was described by Østgaard et al. (2002). To generalize their results, they fitted the precipitation energy to the $A L$ index. While this gives an easily available proxy for the precipitation power, it leads to similar problems related to the $A E$-based proxies as described above. On the other hand, the statistical distribution of electron precipitation can also be measured directly by polarorbiting satellites. For example, Newell et al. (1996) found that the probability of observing accelerated electron precipitation is increased mainly in 18:00-24:00 MLT sector in the nightside. The latitudinal extent of the precipitation depends strongly on the level of magnetic variation. During large storms the oval moves equatorward, while during quiet times the auroral luminosity is concentrated on high latitudes.

There are only a few studies reporting on the energy deposition rate into the ionosphere using global models. Lu et al. (1998) were the first to apply the assimilative mapping of ionospheric electrodynamics (AMIE) technique to estimate the energy deposition rate into the ionosphere during a magnetic storm. The AMIE procedure is based on the mapping procedure by Richmond and Kamide (1988), and it utilizes several models and a variety of measurements in an assimilative way. From the AMIE output Lu et al. (1998) derived the Joule heating and precipitation powers in the ionosphere and concluded that the temporal variation of the Joule heating and precipitation power resembled that of the $A E$ index. $\mathrm{Lu}$ et al. (1998) determined the globally integrated average of Joule heating rate as $190 \mathrm{GW}$ and the average precipitation power as about $90 \mathrm{GW}$ during the particular storm they analyzed.

The global MHD simulations can also be used to investigate the energy flow in the coupled solar windmagnetosphere-ionosphere system. The recent development of the global MHD simulations has focused on the prediction of the magnetospheric state from a given solar wind input, while systematic examination of the magnetospheric response to given solar wind still awaits to be done. Several attempts along this direction have shown to be useful, particularly in cases where the parameters describing the solar wind-magnetosphere-ionosphere coupling are either difficult or impossible to measure globally. For example, the global MHD simulations have been used in mapping the Poynting flux from the solar wind into the magnetosphere to examine the energy flow paths (e.g. Walker et al., 1993; Papadopoulos et al., 1999).

This paper is a continuation of the work by Palmroth et al. (2003), who developed a quantitative method to determine the energy transfer across the magnetopause in a global MHD simulation. Here we calculate the ionospheric energy dissipation, namely the Joule heating and precipitation powers, in the GUMICS-4 global MHD simulation. We determine the latitudinal and longitudinal distributions of the dissipated energy, as well as the temporal variation of the global ionospheric dissipation. We analyze results from two simulated events, a magnetic storm that occurred on 6-7 April 2000, and a substorm that occurred on 15 August 2001. Our final aim is to develop a simple relationship between the solar wind input and ionospheric output. In Sect. 2 we introduce the GUMICS-4 global MHD simulation and the calculation of the ionospheric Joule heating and the precipitation powers; furthermore, we examine theoretically how the ionospheric output depends on solar wind parameters in an ideal MHD. Section 3 describes the observations and the simulation results for the two events. In Sect. 4 we present the results, i.e. the calculated energy dissipation and the latitudinal and longitudinal dissipation distributions for the two events. In Sect. 4.4 we present the fit of the solar wind input data to calculated ionospheric output. Finally, in Sect. 5 we summarize our results and end with a discussion.

\section{Model description}

\subsection{GUMICS-4 global MHD simulation}

GUMICS-4 (Janhunen, 1996) is a global 3-dimensional MHD simulation code that couples the solar windmagnetosphere-ionosphere system in a simulation box with an automatically adaptive Cartesian octogrid.Automatical adaption means that whenever the code detects large gradients the cells near the gradients are divided into 8 daughter cells. The adaptation depends further on location, such that near-Earth cells are more easily refined than, for example, cells at the distant tail. The GUMICS-4 simulation solves the fully conservative MHD equations in the solar wind-magnetosphere domain, whereas electrostatic equations are solved in the ionospheric domain. The simulation box reaches from $X_{\mathrm{GSE}}=32 R_{E}$ upwind to $X_{\mathrm{GSE}}=$ $-224 R_{E}$ in the antisunward direction, and in the $Y_{\mathrm{GSE}}$ and $Z_{\mathrm{GSE}}$ directions to $\pm 64 R_{E}$. The lower limit of the magnetospheric domain is a $3.7 R_{E}$-radius spherical shell from which the field-aligned currents and electron precipitation are mapped to the ionosphere using the dipole field. 
The ionospheric electron density is affected by the solar extreme ultraviolet radiation, as well as the electron precipitation from the magnetosphere, which is assumed to orginate from a Maxwellian source population. The Pedersen and Hall conductivities are computed from the electron density in a three-dimensional grid using 20 non-uniform height levels. The electrostatic potential equation is solved in the ionosphere using the height-integrated conductivities and the field-aligned current from the magnetosphere, after which the ionospheric potential is mapped back to the inner boundary of the magnetosphere and used as a boundary condition for the MHD equations. The spherical ionosphere uses a triangular fixed grid, in which the oval region is more refined (grid resolution of about $100 \mathrm{~km} \times 100 \mathrm{~km}$ ) than, for example, in the equatorial region.

The simulations of the two events were carried out in a code setup similar to that described in Palmroth et al. (2001). In the April 2000 storm simulation the IMF $B_{x}$ was set to zero to ensure the divergence-free input magnetic field. In the 15 August 2001 substorm simulation the IMF $B_{x}$ was set to a constant value of $-3 \mathrm{nT}$, which corresponds to the observed value of IMF $B_{x}$ before 5:00 UT. Consequently, the input magnetic field at the time of onset ( $\sim 4: 30$ UT) was modeled accurately. Of course, constant $B_{x}$ also fulfills the divergence-free condition. In the April 2000 storm simulation the smallest grid size was $0.5 R_{E}$, whereas in the 15 August 2001 substorm simulation the smallest grid size was $0.25 R_{E}$. The denser grid resolution in the magnetosphere typically tends to increase the polar cap potentials, which are typically $20-30 \%$ smaller in GUMICS-4 (with $0.5 R_{E}$ as the smallest grid) than in, for example, SuperDARN observations.

\subsection{Energy dissipated into the ionosphere}

The ionospheric dissipation is calculated as a sum of the power consumed by Joule heating $P_{J H}$ and the precipitating particles. The Joule heating power is calculated as

$P_{J H}=\int \boldsymbol{E} \cdot \boldsymbol{J} d S=\int \Sigma_{P} E^{2} d S$,

where $\boldsymbol{E}$ is the electric field, $\boldsymbol{J}$ the height-integrated current density, $\Sigma_{P}$ the height-integrated Pedersen conductivity, and $d S$ the area element on the spherical ionospheric surface. The quantities are interpolated from the simulation results in an ionospheric grid with a resolution of $1^{\circ}$ in latitude and $3^{\circ}$ in longitude. This interpolation is a necessary operation due to the non-uniform grid utilized by the simulation code, and it does not affect the integration results.

The energy associated with particle precipitation is obtained using formulas given by Robinson et al. (1987), where the height-integrated ionospheric Pedersen and Hall conductivities, $\Sigma_{P}$ and $\Sigma_{H}$, are calculated using the energy flux and the average energy of precipitating electrons. In the present study, we obtain the height-integrated conductivities from the simulation results and analytically invert Eqs. (3) and (4) of Robinson et al. (1987) to obtain the precipitation energy flux.

\subsection{Similarity scaling laws}

Consider the ideal MHD equations written in the primitive variable form

$$
\begin{aligned}
& \partial_{t} \rho=-\nabla \cdot(\rho \boldsymbol{v}) \\
& \rho\left(\partial_{t} \rho+\boldsymbol{v} \cdot \nabla \boldsymbol{v}\right)=-\nabla P+\boldsymbol{j} \times \boldsymbol{B} \\
& \partial_{t} \boldsymbol{B}=\nabla \times(\boldsymbol{v} \times \boldsymbol{B}) \\
& \left(\partial_{t}+\boldsymbol{v} \cdot \nabla\right)\left(P \rho^{-\gamma}\right) 0,
\end{aligned}
$$

where $\boldsymbol{j}=\nabla \times \boldsymbol{B} / \mu_{0}$. Furthermore, let us concentrate on stationary solutions $\left(\partial_{t}=0\right)$, and decompose $\boldsymbol{B}=\boldsymbol{B}_{0}+\boldsymbol{B}_{1}$, where $\boldsymbol{B}_{0}$ is the Earth's internal field and $\boldsymbol{B}_{1}$ is the externally induced part. Then, any solution of Eqs. (2-5) is defined by four functions of three coordinates $\rho(\boldsymbol{x}), \boldsymbol{v}(\boldsymbol{x}), P(\boldsymbol{x})$ and $\boldsymbol{B}_{1}(\boldsymbol{x})$. The similarity scalings of such solutions are new solutions $\rho^{\prime}(\boldsymbol{x}), \boldsymbol{v}^{\prime}(\boldsymbol{x}), P^{\prime}(\boldsymbol{x})$ and $\boldsymbol{B}_{1}^{\prime}(\boldsymbol{x})$ which are related to the original ones by $\rho^{\prime}\left(C_{L} \boldsymbol{x}\right)=C_{\rho} \rho(\boldsymbol{x}), \boldsymbol{v}^{\prime}\left(C_{L} \boldsymbol{x}\right)=$ $C_{v} \boldsymbol{v}(\boldsymbol{x}), P^{\prime}\left(C_{L} \boldsymbol{x}\right)=C_{P} P(\boldsymbol{x})$ and $\boldsymbol{B}_{1}^{\prime}\left(C_{L} \boldsymbol{x}\right)=C_{B_{1}} \boldsymbol{B}_{1}(\boldsymbol{x})$, where $C_{\rho}, C_{v}, C_{P}$ and $C_{B_{1}}$ are the scaling factors for density, velocity, pressure and perturbation magnetic field, and $C_{L}$ is the scaling factor for spatial coordinates. We also introduce a scaling factor $C_{B_{0}}$ for the internal magnetic field in the same way as for the dynamic variables, and require that the new functions satisfy Eqs. (2-5) with $\partial_{t}=0$. In the stationary case, Eqs. (2) and (5) contain only one term and thus do not imply any conditions for the scaling factors, but the momentum equation (3) and Faraday's law (4) read

$\rho \boldsymbol{v} \cdot \nabla \boldsymbol{v}-\frac{1}{\mu_{0}}\left(\nabla \times \boldsymbol{B}_{1}\right) \times \boldsymbol{B}_{0}-\frac{1}{\mu_{0}}\left(\nabla \times \mathbf{B}_{1}\right) \times \boldsymbol{B}_{1}+\nabla P=0(6)$
$\nabla \times\left(\boldsymbol{v} \times \boldsymbol{B}_{0}\right)+\nabla \times\left(\boldsymbol{v} \times \boldsymbol{B}_{1}\right)=0$.

Applying the scalings and requiring that each possible pair of the terms in Eqs. (6) and (7) scales in the same way we obtain the conditions

$C_{\rho} C_{v}^{2}=C_{B_{1}} C_{B_{0}}=C_{B_{1}}^{2}=C_{P}$,

i.e. $C_{B_{0}}=C_{B_{1}}=C_{\rho}^{1 / 2} C_{v}, C_{P}=C_{\rho} C_{v}^{2}$. Requiring that the internal field is the dipole field which scales as $r^{-3}$ we obtain the connection $C_{L}=C_{B_{0}}^{-1 / 3}$ between the coordinate scaling $C_{L}$ and the internal field scaling $C_{B_{0}}$. We thus see that $C_{\rho}$ and $C_{v}$ can be selected freely but the other scaling factors $C_{B_{1}}, C_{B_{0}}, C_{P}$ and $C_{L}$ follow from these two. In other words, by starting from a stationary solution which is valid for solar wind density $\rho$ and velocity $v$, we obtain a two-parameter family of similarity solutions which spans all possible solar wind density and velocity combinations. The nature of the similarity solutions is such that if, for example, the solar wind density is multiplied by 10 , the density and pressure everywhere in the simulation box are also multiplied by 10 , the magnetic fields by $\sqrt{10}$, and the spatial scales by $10^{-1 / 6} \approx 0.68$. The Earth, therefore, becomes larger relative to the magnetosphere, if the solar wind density is increased, but otherwise the MHD solutions are self-similar. 


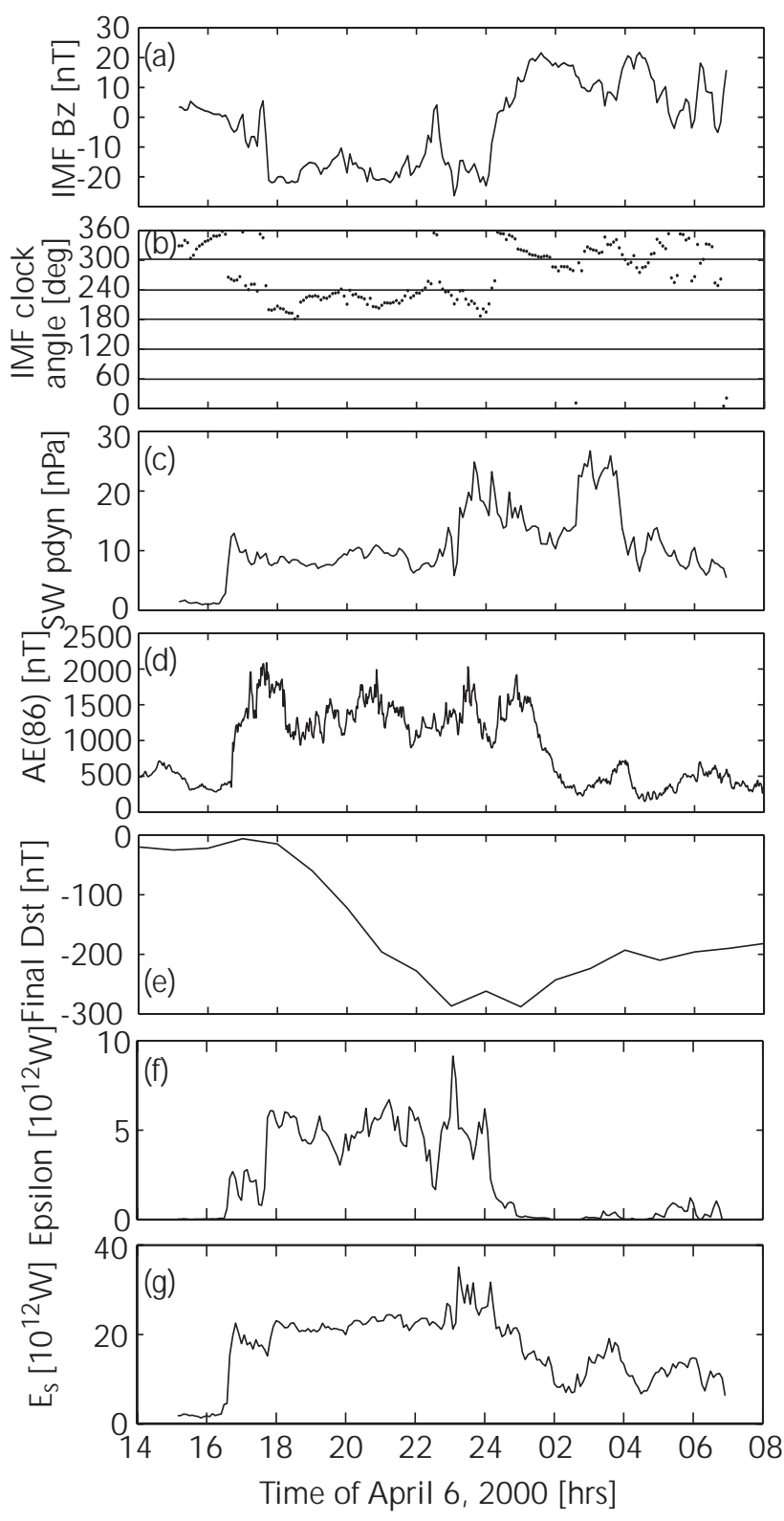

Fig. 1. Solar wind conditions during the April 2000 storm. (a) IMF $B_{z}$, (b) IMF clock angle, (c) solar wind dynamic pressure, (d) $A E$ index measured at 86 stations, (e) final $D_{s t}$ index, (f) $\epsilon$ calculated from the solar wind parameters, and (g) total energy through the magnetopause surface.

Apart from the changing ionospheric feedback and the inherent time-dependence of the solution, the similarity solutions should, therefore, correspond to what we obtain from GUMICS-4.

The ionospheric Joule heating is proportional to the square of the current flowing through the ionosphere, if the ionospheric conductivity pattern and the geometry of the current stay constant. The Joule heating $P_{\mathrm{JH}}$ is given by an ionospheric area integral $P_{\mathrm{JH}}=\int d S J_{P}^{2} / \Sigma_{P}$, where $\Sigma_{P}$ is the height-integrated Pedersen conductivity and $J_{P}$ is the heightintegrated Pedersen current. The MHD similarity solutions scale such that the polar cap expands or shrinks but the current pattern stays approximately self-similar. If the total current $I$ flowing through the ionosphere is kept constant, $J_{P}$ is proportional to $I / R_{P C}$, where $R_{P C}$ is the polar cap radius. Since the polar cap area is proportional to $R_{P C}^{2}$, the Joule heating $P_{\mathrm{JH}}$ is independent of $R_{P C}$. Thus, we conclude that, approximately, $P_{\mathrm{JH}}$ is proportional to $I^{2}$. The total current $I$ flowing through the ionosphere scales in the same way as the magnetospheric current systems, thus we obtain $I \sim j L^{2}$, where the current density $j \sim B_{1} / L$ and $L$ is the spatial length, i.e. $I \sim\left(B_{1} / L\right) L^{2}=B_{1} L \sim P^{1 / 2} P^{-1 / 6}=$ $P^{1 / 3}$, where $P$ is the solar wind dynamic pressure. Thus, $P_{\mathrm{JH}} \sim P^{2 / 3} \sim\left(\rho v^{2}\right)^{2 / 3} \sim \rho^{2 / 3} v^{4 / 3}$.

The ionospheric particle precipitation energy flux per unit area from a magnetospheric Maxwellian source plasma is proportional to $P_{t h} v_{t h}$, where $P_{t h}$ is the thermal pressure of the source plasma and $v_{t h} \sim v$ its thermal velocity (Janhunen and Olsson, 1998). Thus, the total power of particle precipitation $P_{\text {prec }}$ scales as $P_{\text {prec }} \sim P_{t h} v_{t h} A_{\mathrm{PC}} \sim \rho v^{3} A_{\mathrm{PC}}$, where $A_{\text {PC }}$ is the polar cap area. In a dipole field a simple consideration shows that $A_{\mathrm{PC}} \sim L^{-1} \sim P^{1 / 6} \sim\left(\rho v^{2}\right)^{1 / 6}$ and thus $P_{\text {prec }} \sim \rho^{7 / 6} v^{10 / 3}$.

To summarize, we have obtained that

$$
\begin{aligned}
& P_{\mathrm{JH}} \sim \rho^{2 / 3} v^{4 / 3} \\
& P_{\text {prec }} \sim \rho^{7 / 6} v^{10 / 3} \\
& I \sim P^{1 / 3}
\end{aligned}
$$

where $\rho$ and $v$ are the solar wind density and velocity, respectively, $P=\rho v^{2}$ is the dynamic pressure, and $P_{\mathrm{JH}}$ and $P_{\text {prec }}$ are the total Joule heating and particle precipitation powers, respectively.

\section{Event descriptions}

\section{$3.1 \quad 6-7$ April 2000 storm}

Figure 1 presents the 6-7 April 2000 storm observations, as well as the energy input to the magnetosphere using GUMICS-4 simulation and an empirical parameter. Figures $1 \mathrm{a}-\mathrm{e}$ show, respectively, the IMF $B_{z}$ component, the IMF clock angle, the solar wind dynamic pressure, the $A E$ index computed from 86 stations, and the final $D_{s t}$ index. Figure 1a shows that the IMF $B_{z}$ turned strongly southward at $\sim$ 18:00 UT on 6 April 2000, and rotated strongly northward at $\sim 00: 00$ UT on 7 April 2000. During the storm main phase (18:00-24:00 UT), the IMF clock angle was in the sector between $180^{\circ}$ and $240^{\circ}$ (Fig. 1b). The solar wind dynamic pressure (Fig. 1c) was unusually high throughout the event, reaching almost $30 \mathrm{nPa}$ during the storm recovery phase at 7 April 2000. The $A E$ index (Fig. 1d) was strongly enhanced, being almost steadily over $1500 \mathrm{nT}$ during the storm. The final $D_{s t}$ index (Fig. 1e) decreased close to $-300 \mathrm{nT}$ at storm maximum. (For more details, see Huttunen et al., 2002). 
Panels 1f and 1g depict the April 2000 storm energetics using two different approaches. The $\epsilon$ parameter (Fig. 1f) (Akasofu, 1981), which represents the energy input into the inner magnetosphere, enhances to approximately half of its maximum at the storm sudden commencement (SSC) at 16:40 UT and reaches its maximum later during the storm main phase. The energy input stops when the IMF $B_{z}$ turns northward. Panel $1 \mathrm{~g}$ shows the total energy transferred through the magnetopause as evaluated from the GUMICS-4 global MHD simulation (Palmroth et al., 2003). In the simulation, the energy input also starts at the SSC, but increases immediately to values characteristic of the main phase, and does not decrease to zero when the IMF turns northward. This can be attributed to the fact that the energy input is also dependent on the solar wind dynamic pressure (Scurry and Russell, 1991), a factor that is highly enhanced during the event and is present in the $\epsilon$ equation only through the solar wind bulk speed. A relative error analysis (not shown) indicated that the relative error of the energy input on 5\% larger and smaller surfaces compared to the net input energy is small during the storm main phase, whereas fluctuations appear at the SSC (around 16:00 UT) and during the recovery phase (00:00-07:00 UT on 7 April 2000). The fluctuations of the relative error are most probably due to the surface motion. Owing to the continuous forcing of the solar wind during the main phase the surface is more stationary than during the recovery phase.

\subsection{August 2001 substorm}

Figure 2 shows the 15 August 2001 substorm observations and the energy transfer rates calculated as above. The solar wind measurements were recorded by the Geotail spacecraft. Panels $2 \mathrm{a}-\mathrm{c}$ show the IMF $B_{z}$, IMF clock angle, and the solar wind dynamic pressure. The substorm occurred when the North American sector was in the nightside, therefore, panel $2 \mathrm{~d}$ presents the auroral electrojet index calculated from the CANOPUS magnetometer array. Ten stations (FCHU, CONT, DAWS, ESKI, GILL, ISLL, MCMU, RANK, RABB, FSIM) were used, from which the minimum of the north component was selected at each time step, yielding the $C L$ index. Panels $2 \mathrm{e}$ and $2 \mathrm{f}$ show the $\epsilon$ parameter calculated from the solar wind parameters, and the total energy transferred through the magnetopause surface in the GUMICS4 MHD simulation using the method described in Palmroth et al. (2003).

Figure $2 \mathrm{a}$ shows that the IMF $B_{z}$ was around zero at the beginning of the simulated time period and turned weakly southward $\sim 03: 39$ UT. Simultaneously, the IMF clock angle (Fig. 2b) rotated into the sector $120^{\circ}-180^{\circ}$. Solar wind dynamic pressure (Fig. 2c) was low, below $1 \mathrm{nPa}$, during the event. The onset of a modest substorm $(\sim-500 \mathrm{nT})$ occurred at 04:27 UT in the CANOPUS magnetograms (Fig. 2d). Thus, the growth phase of the substorm lasted $48 \mathrm{~min}$, and at substorm onset the IMF was still southward, indicating that the energy input mechanism was still active. The $\epsilon$ parameter (Fig. 2e) started to increase when the IMF

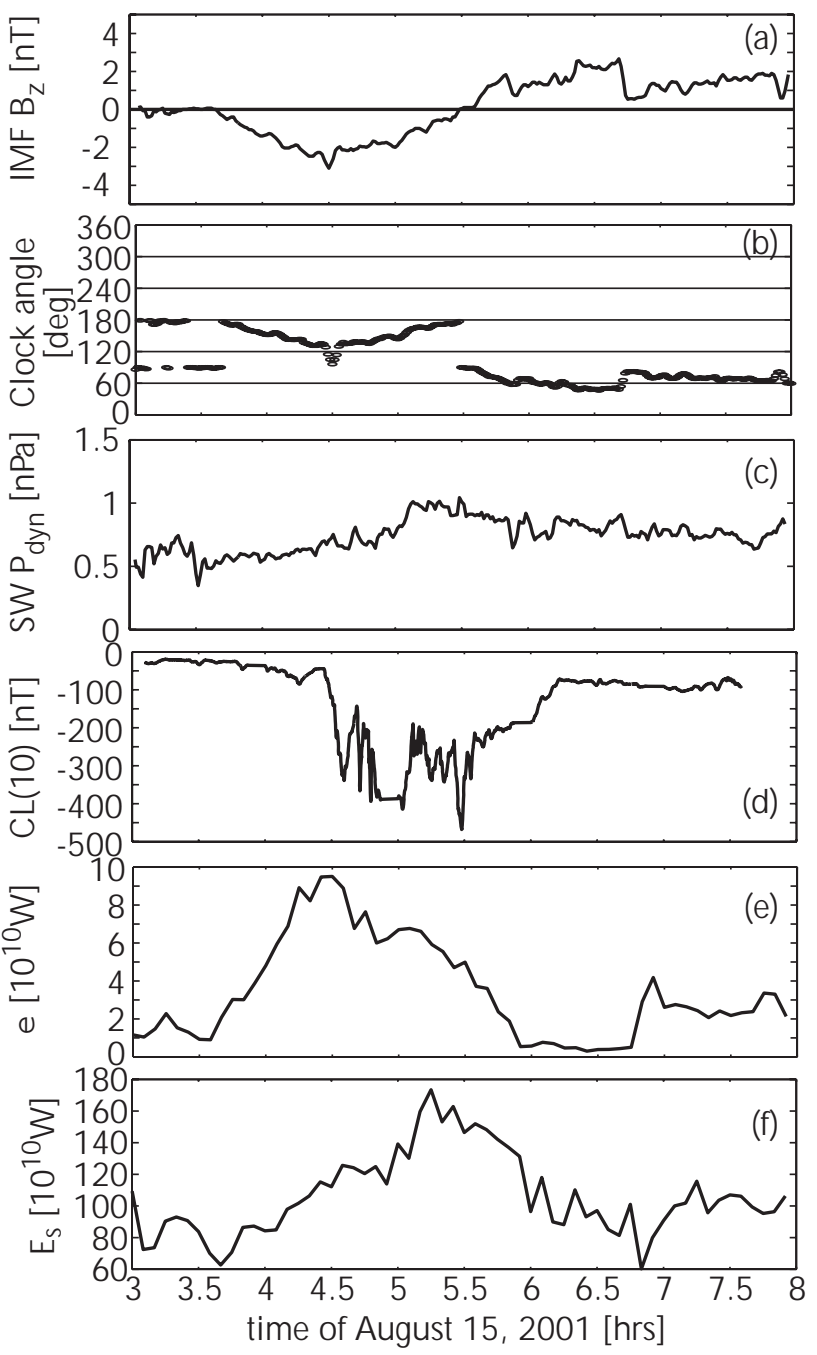

Fig. 2. Solar wind conditions during the 15 August 2001 substorm. (a) IMF $B_{z}$, (b) IMF clock angle, (c) solar wind dynamic pressure, (d) $C L$ index calculated from CANOPUS magnetometer network, (e) $\epsilon$, (f) total energy through the magnetopause surface in a global MHD simulation.

$B_{z}$ turned southward. The $\epsilon$ parameter reached a quite moderate peak value of $1 \cdot 10^{11} \mathrm{~W}$ simultaneously with the minimum of IMF $B_{z}$. The total energy transferred through the MHD magnetopause started to increase about a half an hour later than $\epsilon$, and increased until the IMF $B_{z}$ rotated northward.

As seen from the GUMICS-4 simulation (not shown), the tail stretched until 5:00 UT, which was the time of the onset in the simulation. The expansion phase lasted until 05:40 UT, and the recovery phase took $25 \mathrm{~min}$. Comparing to Fig. 2d, the simulation onset was about a half an hour later than the observed onset, and the simulation substorm was a half an hour faster than the observed substorm, as they both recovered about the same time. 

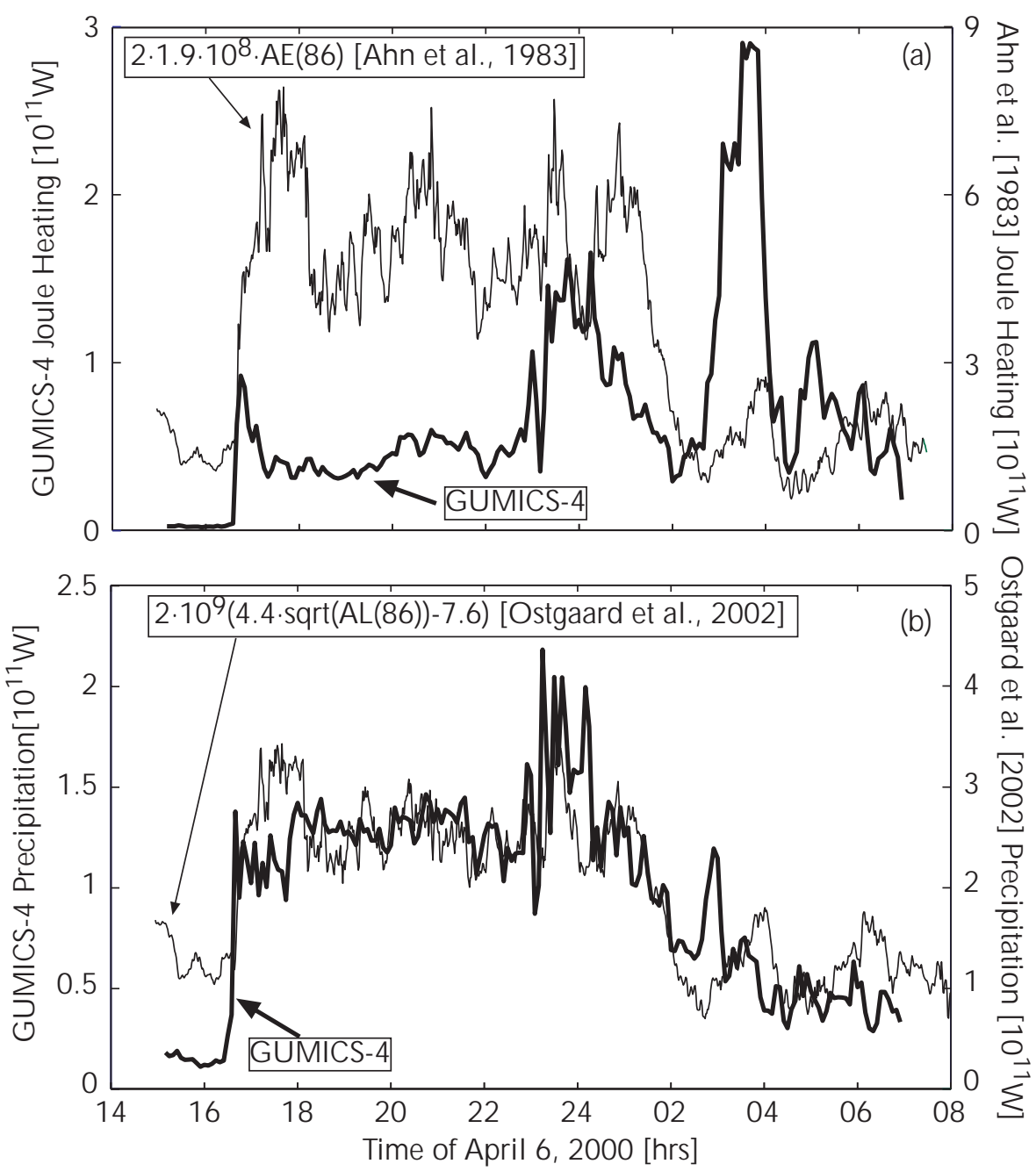

Fig. 3. (a) Joule heating power and (b) precipitation power in the ionosphere during the April 2000 storm as described by the GUMICS-4 global MHD simulation (thick line). The thin lines are calculated using equations presented in Ahn et al. (1983) and Østgaard et al. (2002).

\section{Results}

\subsection{Ionospheric dissipation}

Figures $3 \mathrm{a}$ and $\mathrm{b}$ present the ionospheric Joule heating power and precipitation power in the April 2000 storm. Thick lines show the Joule heating and the precipitation power calculated from the simulation, whereas thin lines depict the Joule heating and the precipitation power using the Ahn et al. (1983) and Østgaard et al. (2002) proxies, respectively. The left vertical axis is for the GUMICS-4 results, whereas the right vertical axis is for the Ahn et al. (1983) and Østgaard et al. (2002) formulas. The Østgaard et al. (2002) method is based on Polar satellite measurements of particle precipitation fitted to $A E$ and $A L$ indices, while Ahn et al. (1983) used an empirical method based on ground magnetic field measurements to calculate the Joule heating rates and fitted the results to $A E$ and $A L$ indices. The Ahn et al. (1983) and Østgaard et al. (2002) proxies are multiplied by two, to account for ionospheric dissipation in both hemispheres. Note that the precipitation power computed from the simulation is calculated from $60^{\circ}$ latitude poleward, because latitudes below $60^{\circ}$ do not reach the $3.7 R_{E}$ shell (the inner boundary of the magnetospheric domain), and thus there cannot be any magnetospheric precipitation sources below this latitude in the simulation.

As shown in Fig. 3, in the simulation at the SSC onset $\left(\sim 16: 40\right.$ UT), the precipitation power $\left(\sim 10 \cdot 10^{10} \mathrm{~W}\right)$ slightly exceeds the Joule heating power $\left(\sim 7 \cdot 10^{10} \mathrm{~W}\right)$. After the SSC, the Joule heating power slightly decreases, but the precipitation power stays at the same level. Comparison with Fig. 1 shows that the temporal variation of Joule heating resembles the temporal variation of the solar wind dynamic pressure, whereas the precipitation power has a completely different shape. The precipitation power starts to decrease at the end of the storm main phase, while the Joule heating reaches its largest value during the recovery phase during a large peak in the solar wind dynamic pressure. Otherwise, the amount of power dissipated by the precipitation and the Joule heating are roughly comparable, with precipitation power showing 

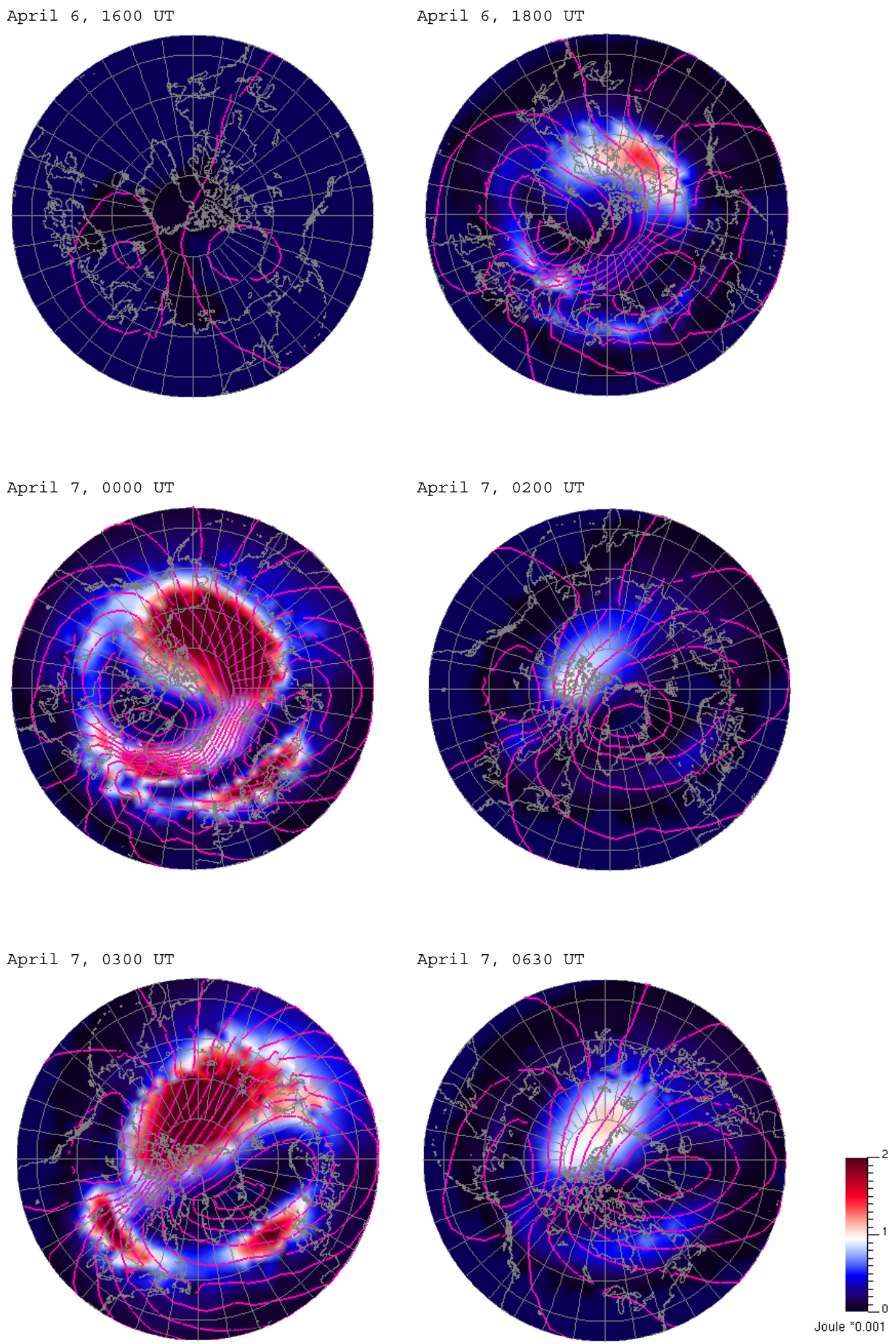

Fig. 4. Joule heating power color-coded in the simulation at six moments of time during the April $2000 \mathrm{storm}^{\mathrm{units}} \mathrm{Wm} \mathrm{m}^{-2}$. 
Table 1. Summary of the power dissipated into the ionosphere during the April 2000 storm.

\begin{tabular}{lll}
\hline$\left[10^{10} \mathrm{~W}\right]$ & $<$ power $>$ & $\max ($ power $)$ \\
\hline Joule heating & $6.9(42 \%)$ & $29.1(57 \%)$ \\
Precipitation & $9.5(58 \%)$ & $21.8(43 \%)$ \\
\hline
\end{tabular}

less time variability than the Joule heating power. Table 1 summarises the average and peak values of dissipated Joule heating and precipitation powers, and the relative contributions are also shown.

In Fig. 3a the Joule heating calculated using the Ahn et al. (1983) method does not compare well with the Joule heating calculated from the global MHD simulation. The average level during the storm main phase $\left(\sim 5 \cdot 10^{11} \mathrm{~W}\right)$ is about ten times larger than the Joule heating rate in the simulation. Also, the temporal variation of the two curves are different. While the Joule heating in the simulation appears to be correlated with the solar wind dynamic pressure, the Ahn et al. (1983) proxy has (by definition) the shape of the $A E$ index. However, in Fig. $3 b$ the precipitation power calculated from the Østgaard et al. (2002) proxy and the precipitation power calculated from the simulation have similar temporal variation, only the precipitation power in the Østgaard et al. (2002) proxy is two times larger than the precipitation power in the simulation. The Østgaard et al. (2002) proxy starts at a higher level before the storm SSC, otherwise the temporal variation of the two curves are remarkably similar.

Figure 4 shows the Joule heating color-coded in the simulation during the April 2000 storm in units of $\mathrm{Wm}^{-2}$. The pink lines show the potential isocontours with $10 \mathrm{kV}$ spacing. The local noon is at the top, 18:00 MLT to the left, 24:00 MLT at the bottom and 06:00 MLT to the right of each plate. Before the storm SSC (16:00 UT) the ionosphere is quiet. At 18:00 UT during the main phase, the polar cap potential difference has increased. Also, the Joule heating is enhanced, with its maximum clearly on the dayside. Enhanced Joule heating also occurred within the polar cap in the region where the electric field is largest (potential contours are close to each other). Furthermore, there is a faint maximum in the midnight sector along the oval. At 00:00 UT, all regions show much enhanced Joule heating power, and the potential difference has further increased. At 02:00 UT the Joule heating power has decreased with only a small distribution over the polar cap. At 03:00 UT, at the largest peak in the Joule heating power rate during the event (during the largest pressure pulse), the Joule heating power distribution covers both the dayside and the nightside ovals, as well as regions within the polar cap. At 06:30 UT, near the end of the simulated period, the decreased Joule heating rate is concentrated within the polar cap; the oval shows only faintly.

Figure 5 shows the precipitation power $\left[\mathrm{Wm}^{-2}\right]$ colorcoded at the same moments of time as in Fig. 4. The outermost circle is the latitude $60^{\circ}$ in the ionosphere, and the innermost circle is $88^{\circ}$ in latitude, and MLT sectors are as in Fig. 4. Before the storm (16:00 UT) there is no significant precipitation into the ionosphere. At 18:00 UT, the precipitation power has increased with a clear maximum in the dayside in the cusp region. At 00:00 UT the situation has not changed from the previous panel, but at 02:00 UT the dayside maximum has been diminished, and instead, particularly in the nightside and dawn oval, there are clear precipitation power maxima. At 03:00 UT, the precipitation has increased in the oval region, but at 06:30 UT the precipitation power rate has decreased almost to the level preceding the storm.

The total ionospheric dissipation can be compared to the total energy transferred through the magnetopause surface (see Figs. 1 and 2). As can be seen from Fig. 1, the energy input through the magnetopause surface during the main phase of the April 2000 storm is $\sim 25000 \mathrm{GW}$, whereas the $\epsilon$ parameter suggests an energy input of $\sim 5000 \mathrm{GW}$ during the main phase. The ionosphere consumes the total amount of $\sim 190 \mathrm{GW}$ during the main phase, which is less than $1 \%$ of the energy transferred through the surface and $\sim 4 \%$ of $\epsilon$. During the recovery phase, $\sim 10000 \mathrm{GW}$ is transferred through the magnetopause surface, and the total amount of $\sim 170 \mathrm{GW}$, about $2 \%$ of input, is dissipated into the ionosphere.

Figure 6 presents the ionospheric Joule heating power and the precipitation power during the 15 August 2001 substorm simulation; the format of the figure is similar to that in Fig. 3. The Joule heating and precipitation powers start to increase around 04:00 UT, reaching their peaks around 05:30 UT. The decreasing phase of the ionospheric dissipation lasts until $\sim 06: 20$ UT. Both ionospheric dissipation power rates show an increasing trend during the simulated time period. The peak value of the ionospheric Joule heating rate is about $50 \%$ of the precipitation energy peak value. Table 2 summarises the ionospheric dissipation power as average and maximum values; also the relative contributions are shown.

For the substorm simulation case, the temporal variation of the Joule heating and precipitation powers from the Ahn et al. (1983) and the Østgaard et al. (2002) proxies appear to be remarkably similar with the temporal variation of the Joule heating and the precipitation powers calculated from the simulation. Note, however, that the scales between the left and right vertical axes are not the same. The Joule heating in the Ahn et al. (1983) proxy is over thirty times larger than the Joule heating in the simulation. The precipitation in the Østgaard et al. (2002) proxy is about ten times larger than the precipitation power in the simulation. Comparing to Fig. 2, during the 15 August 2001 substorm, on average, $1200 \mathrm{GW}$ is transferred through the magnetopause surface during the substorm, while the $\epsilon$ parameter indicates about $50 \mathrm{GW}$ energy input on average. The ionosphere consumes only $12 \mathrm{GW}$, on average (Table 2 ), which is $\sim 1 \%$ of the total transferred energy, and $\sim 24 \%$ of the average $\epsilon$ during the substorm.

Figure 7 presents the Joule heating at six instants of time during the August 2001 substorm simulation, the format of the figure is otherwise the same as in Fig. 4 but the color scal- 
April 6, 1600 UT

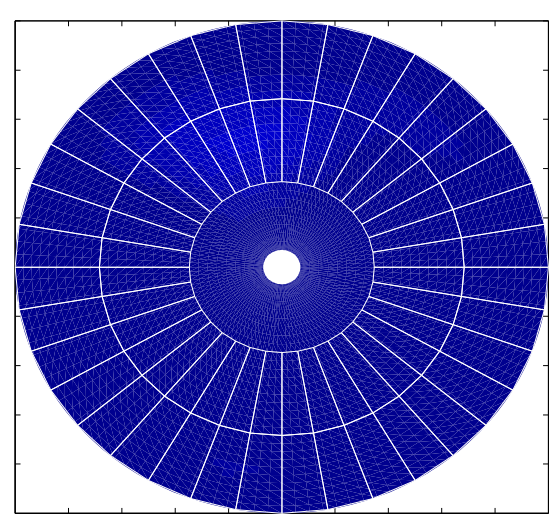

April 7, 0000 UT
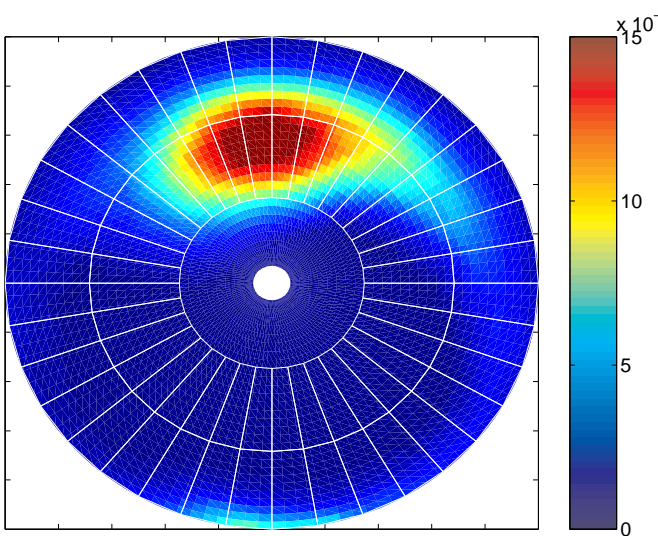

April 7, 0300 UT

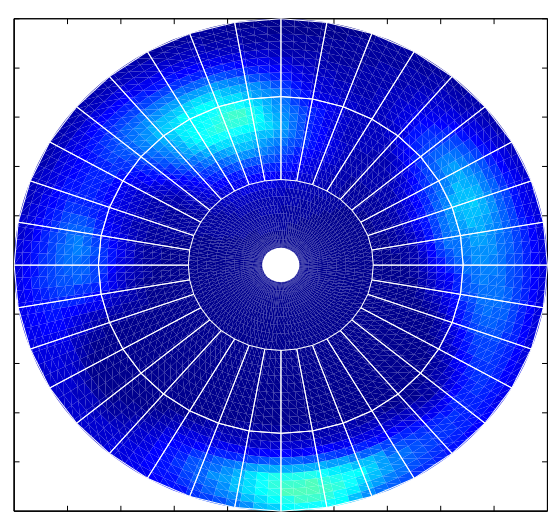

April 6, 1800 UT
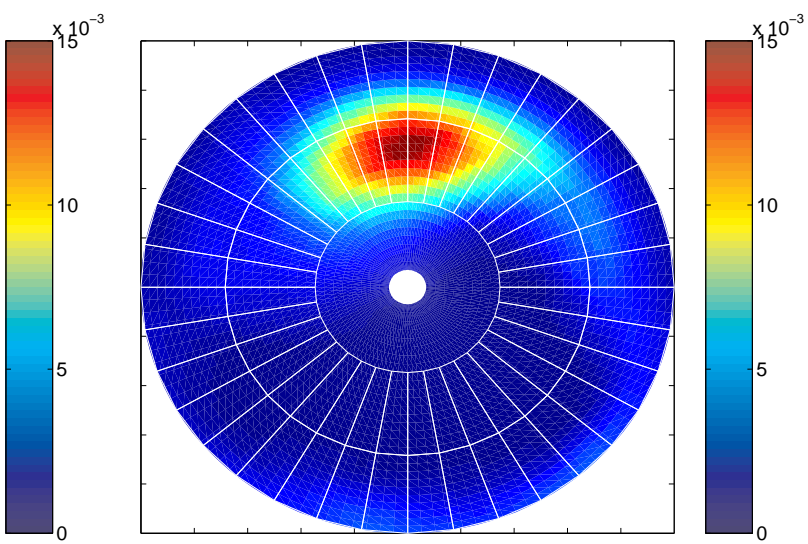

April 7, 0200 UT
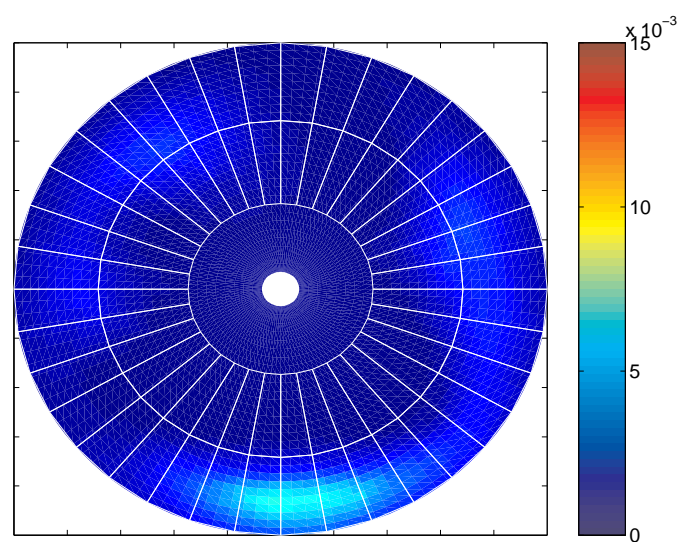

April 7, 0630 UT
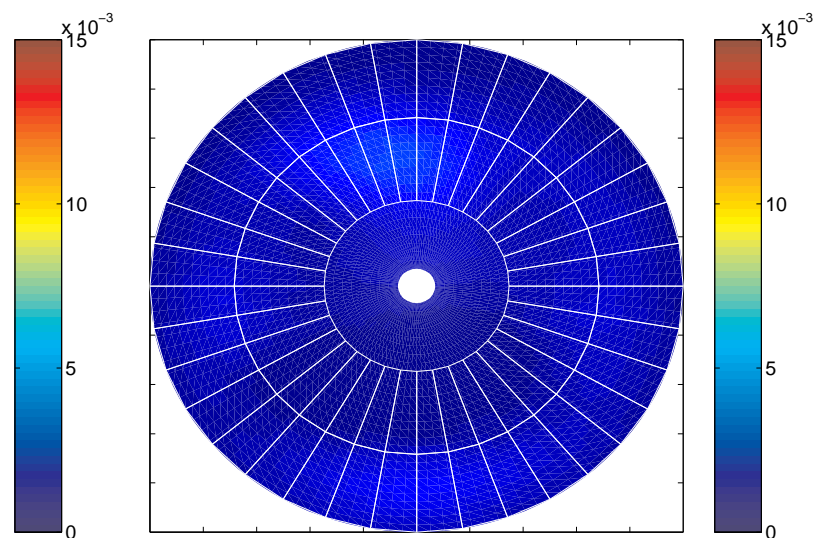

Fig. 5. Precipitation power color-coded in the simulation at six moments of time during the April $2000 \mathrm{storm} \mathrm{units}_{\mathrm{Wm}}{ }^{-2}$.

ing is different. Before the substorm onset (05:00 UT in the simulation) a small amount of Joule heating is concentrated mainly in the nightside oval. At 05:30 UT, after the onset, the Joule heating power rate is enhanced in the nightside oval, and there is a small maximum in the duskside oval. Half an hour later the Joule heating power has already decreased to the level preceding the substorm.

Figure 8 shows the precipitation color-coded in the simulation during the same time instant as above in Fig. 7, and the format of the figure is similar to Fig. 5 except for the 

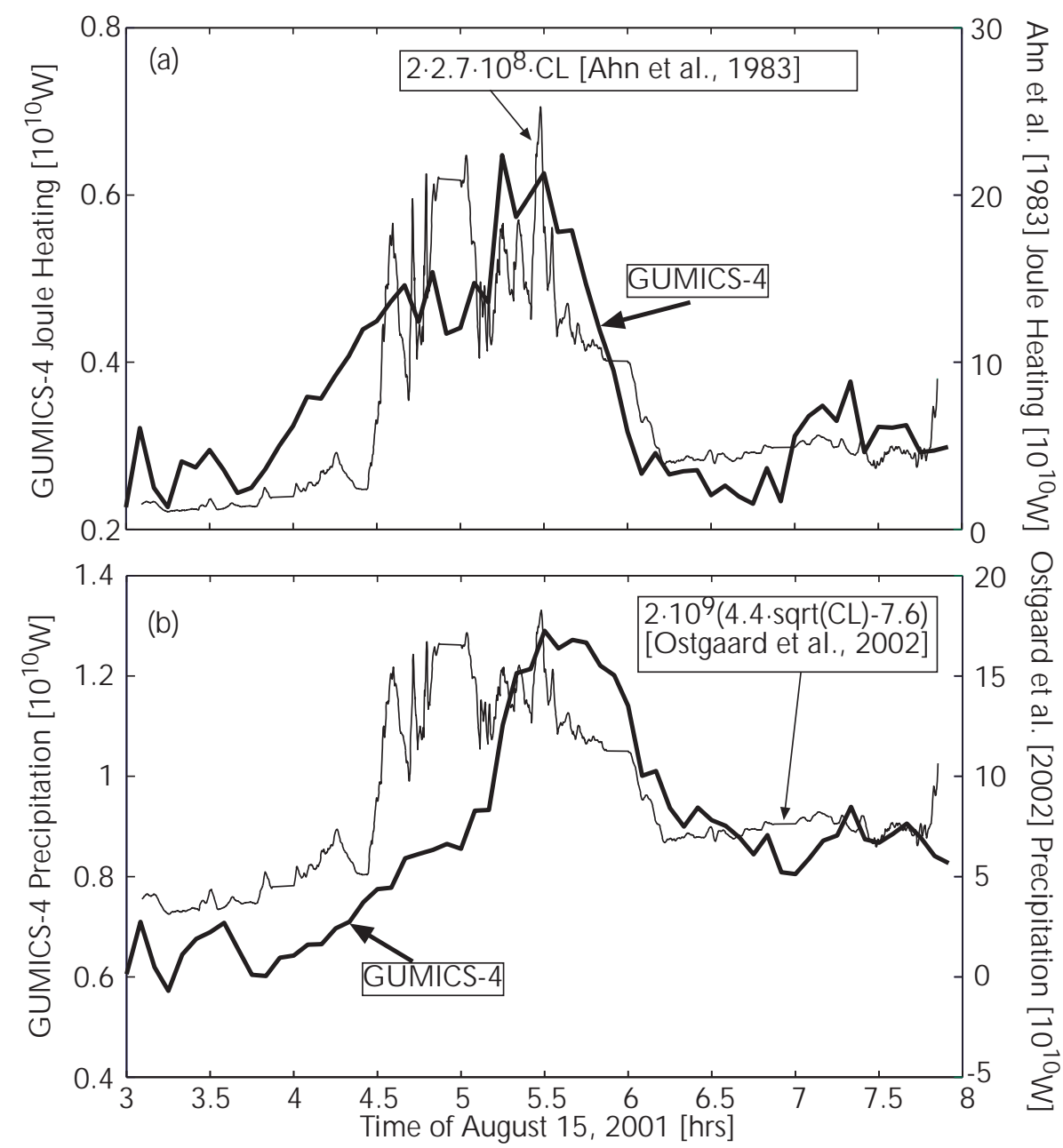

Fig. 6. (a) Joule heating power and (b) precipitation power in the ionosphere during the 15 August 2001 substorm as described by the GUMICS-4 global MHD simulation (thick line). The thin lines are calculated using equations presented in Ahn et al. (1983) and Østgaard et al. (2002).

Table 2. Summary of the power dissipated into the ionosphere during the 15 August 2001 substorm.

\begin{tabular}{lll}
\hline$\left[10^{9} \mathrm{~W}\right]$ & $<$ power $>$ & $\max ($ power $)$ \\
\hline Joule heating & $3.6(29 \%)$ & $6.5(33 \%)$ \\
Precipitation & $8.7(71 \%)$ & $12.9(67 \%)$ \\
\hline
\end{tabular}

color scaling. Before the substorm onset there is already a small amount of precipitation centered approximately at the 21:00 MLT sector, and a smaller maximum exists approximately at the 02:00 MLT sector. After the onset the precipitation maximum at the 21:00 MLT sector is enhanced, persisting still at 06:00 UT. At 07:00 UT the precipitation power has recovered to the level preceding the substorm. The 02:00 MLT maximum remains approximately the same in size throughout the simulated period.

\subsection{Joule heating power distribution in the ionosphere}

Figure 9 presents the ionospheric Joule heating rates distributed at different longitudes and latitudes during the April 2000 storm simulation: Figs. 9a-c show the Joule heating rates in the dayside, dawn and dusk sectors, and in the nightside, respectively. Figures $9 \mathrm{~d}-\mathrm{f}$ show the Joule heating rates at low latitudes, within the auroral oval, and within the polar cap, respectively. As is evident from Fig. 9, the SSC is visible at all local times and latitudes simultaneously around the ionosphere. The temporal variation of the different curves are similar, indicating that all the peaks and valleys in the Joule heating power occur at the same time. However, Fig. 9 also shows that the location contributing mostly to the Joule heating rate during the storm evolution is the dayside oval and low latitudes. For instance, the nightside Joule heating rate is about $40 \%$ of the dayside Joule heating rate.

Figures 10a-f present the Joule heating power distribution during the 15 August 2001 substorm simulation in the dayside, dawn and dusk sectors, nightside, low latitudes, oval 

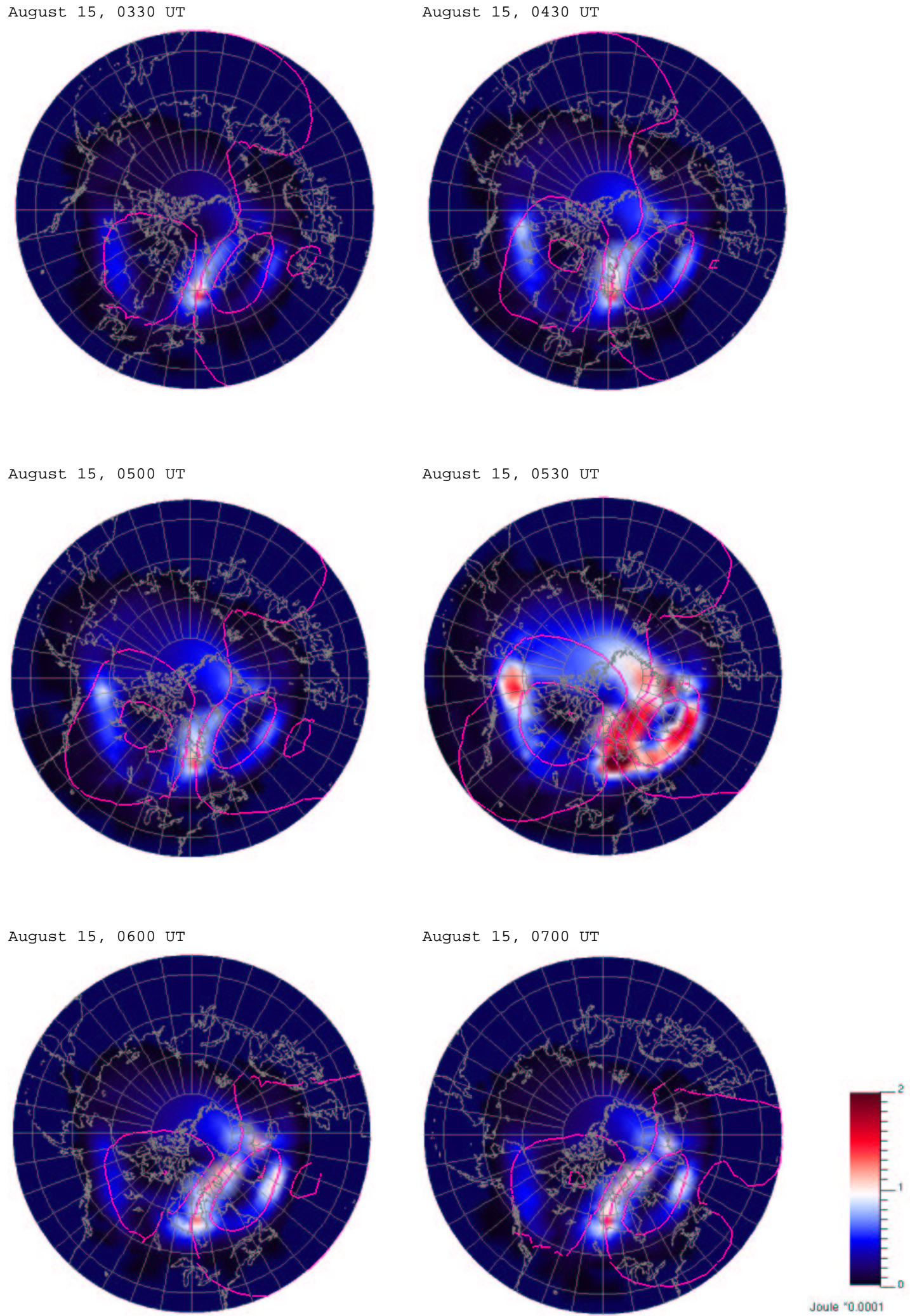

Fig. 7. Joule heating power color-coded in the simulation at six moments of time during the August $2001 \mathrm{substorm}^{\mathrm{units}} \mathrm{Wm} \mathrm{m}^{-2}$. 
August 15, 0330 UT

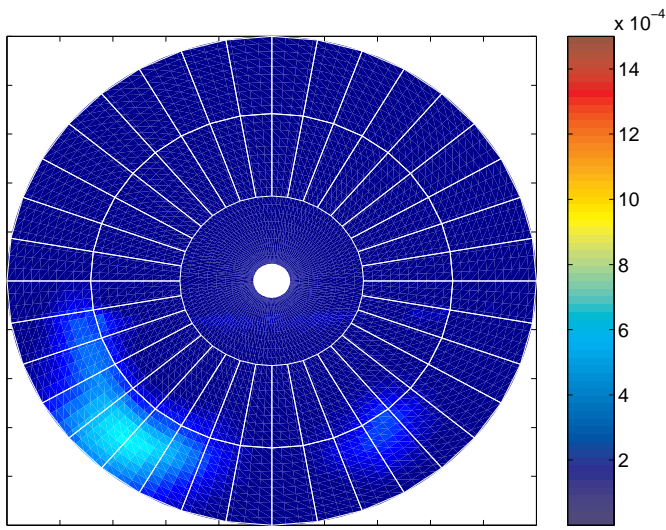

August 15, 0500 UT
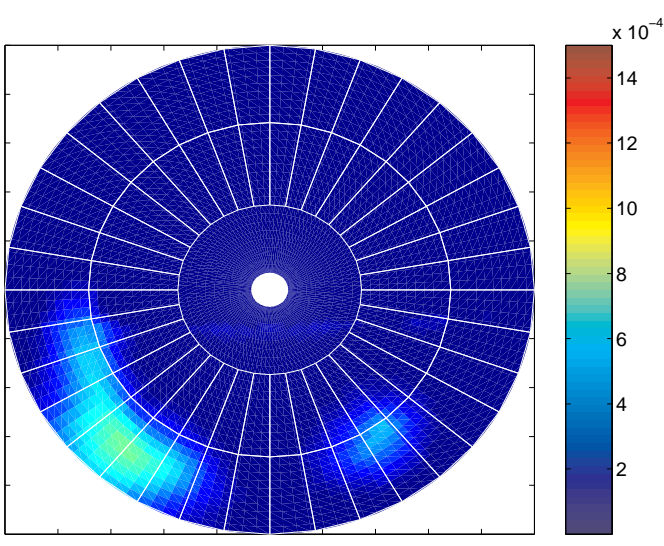

August 15, 0600 UT
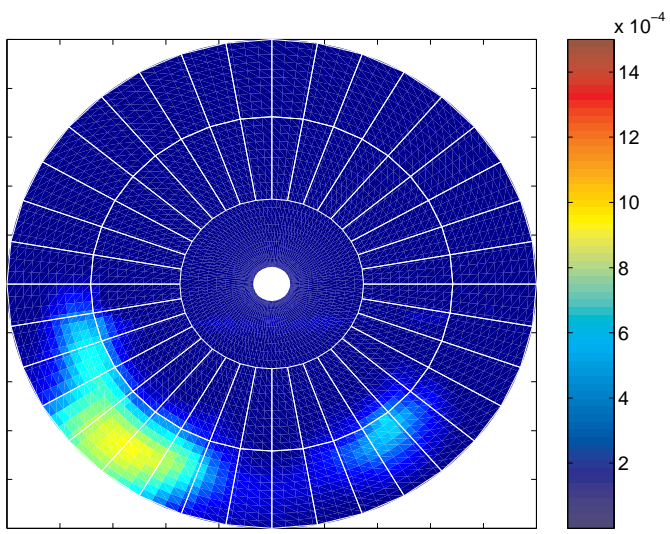

August 15, 0430 UT

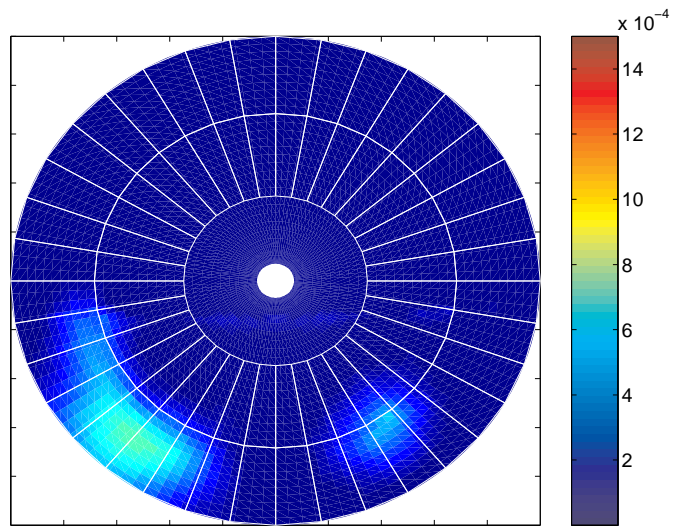

August 15, 0530 UT

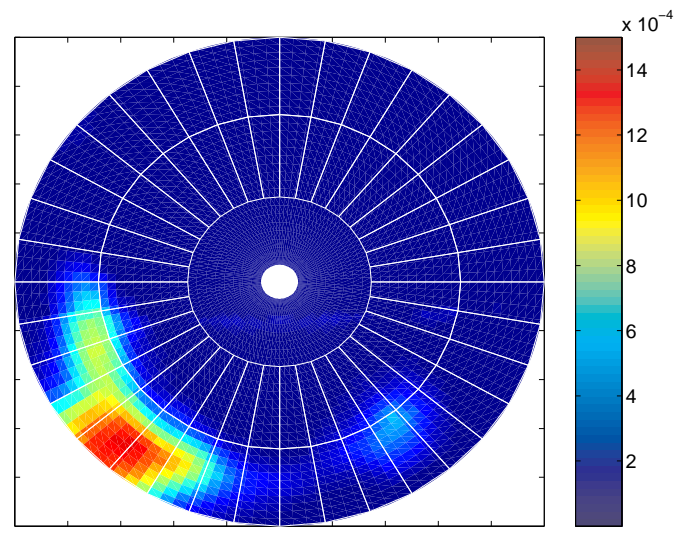

August 15, 0700 UT

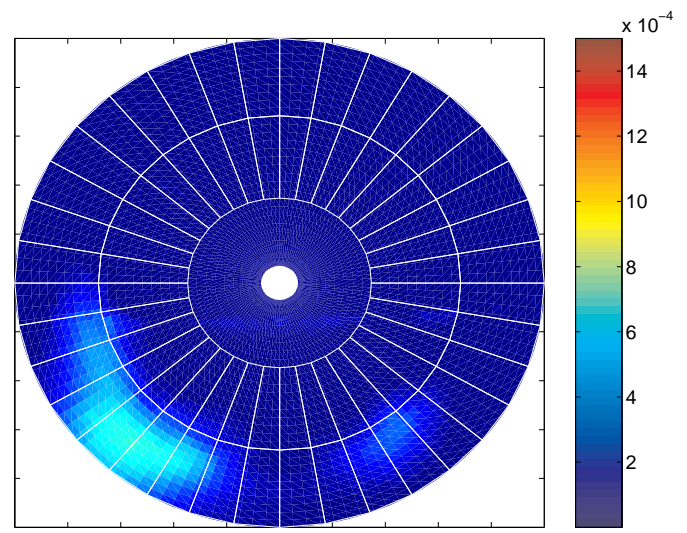

Fig. 8. Precipitation power color-coded in the simulation at six moments of time during the August 2001 substorm, units $\mathrm{Wm}^{-2}$.

area, and in the polar cap, respectively. Contrary to the storm event, in the substorm case most of the Joule heating power is concentrated to the oval latitudes. The Joule heating power in the nightside exceeds the Joule heating power in the dawn and dusk and in the dayside.

\subsection{Precipitation power distribution in the ionosphere}

Figure 11 presents the energy associated with the precipitation in the dayside, dawn and dusk sectors, the nightside (Figs. 11a-c), and in the oval and polar cap areas (Figs. 11d- 

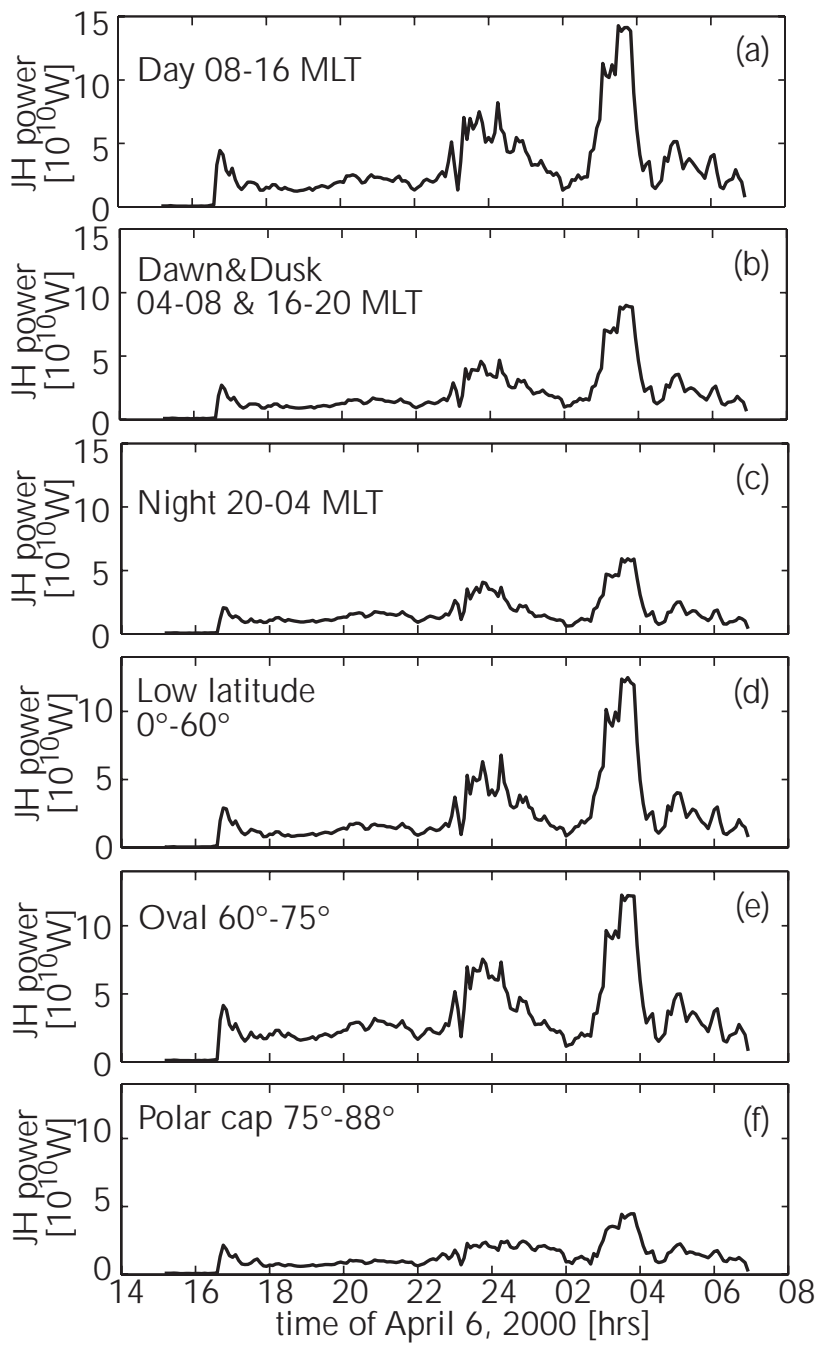

Fig. 9. Joule heating power during the April 2000 storm in (a) the dayside 08:00-16:00 MLT, (b) dawn 04:00-08:00 MLT and dusk 16:00-20:00 MLT, (c) nightside 20:00-04:00 MLT, (d) low latitudes $0^{\circ}-60^{\circ}$, (e) oval latitudes $60^{\circ}-75^{\circ}$, (f) polar cap latitudes $75^{\circ}-88^{\circ}$. Both hemispheres are present in panels.

e) in the April 2000 storm simulation. Figure 11 clearly illustrates that the major part of precipitation power is dissipated in the dayside oval and cusp regions during the April 2000 storm simulation.

Figure 12 shows the precipitation power distributed over latitude and longitude during the 15 August 2001 substorm simulation. Figures $12 \mathrm{a}-\mathrm{c}$ show the precipitation powers in the dayside, dawn and dusk sectors, and in the nightside, whereas Figs. 12d and e depict the precipitation powers in the oval and polar cap areas, plotted in the same scale. Figure 12 demonstrates that during the substorm, a major part of the precipitation power comes from the nightside oval. The dawn and dusk precipitation peak value reaches $\sim 80 \%$ of the nightside precipitation peak value.

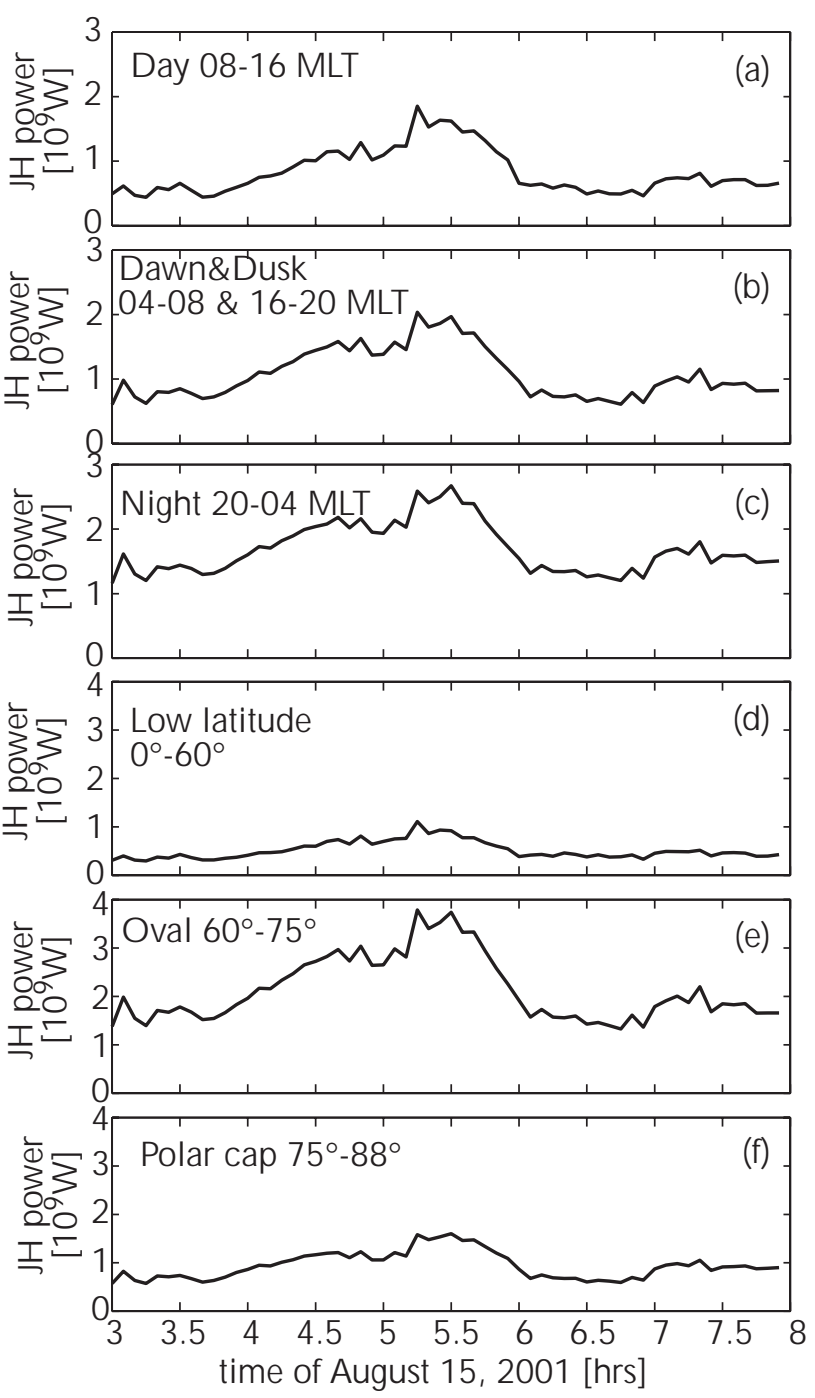

Fig. 10. Joule heating power during the 15 August 2001 substorm in the (a) dayside 08:00-16:00 MLT, (b) dawn 04:00-08:00 MLT and dusk 16:00-20:00 MLT, (c) nightside 20:00-04:00 MLT, (d) low latitudes $0^{\circ}-60^{\circ}$, (e) oval latitudes $60^{\circ}-75^{\circ}$, and (f) polar cap latitudes $75^{\circ}-88^{\circ}$. Both hemispheres are present in panels.

\subsection{Relation between input and output}

After calculating the ionospheric dissipation directly from the simulation of the two events, we set out to search for a formulation of the ionospheric dissipation as a function of the solar wind parameters. Ideally one would like to find a general formulation, which gives the same functional dependence on the solar wind parameters for both simulated events, implying that it may be valid also in a more general case. The parameters to be considered include at least the solar wind density $\rho$, velocity $v$ and the IMF $B_{z}$. We chose the simplest power law function for fitting the simulation data, i.e.

$P_{\text {ionosphere }}=C\left(\frac{\rho}{\rho_{0}}\right)^{a}\left(\frac{v}{v_{0}}\right)^{b}\left[\exp \left(\frac{B_{z, I M F}}{\sqrt{2 \mu_{0} p_{d y n}}}\right)\right]^{d}$, 


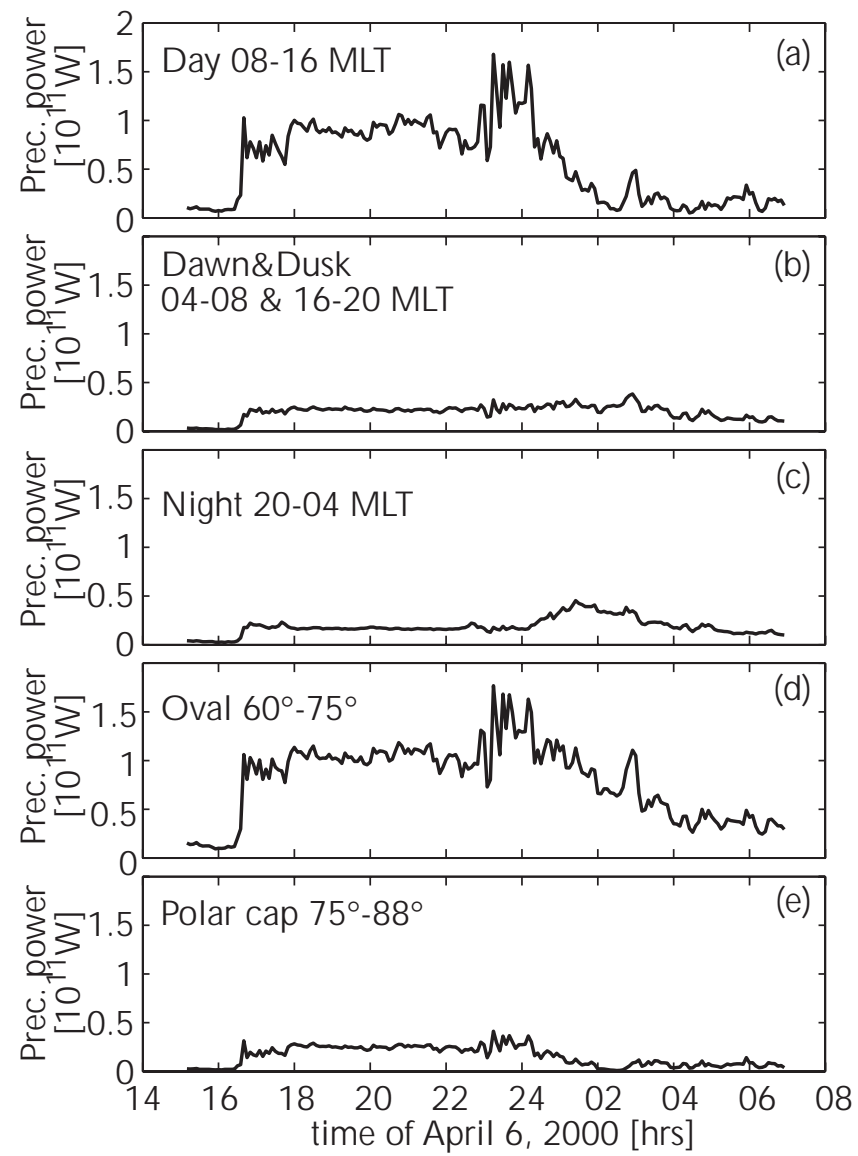

Fig. 11. Precipitation energy during the April 2000 storm in (a) the dayside 08:00-16:00 MLT, (b) dawn 04:00-08:00 MLT and dusk 16:00-20:00 MLT, (c) nightside 20:00-04:00 MLT, (d) oval latitudes $60^{\circ}-75^{\circ}$, and (e) polar cap latitudes $75^{\circ}-88^{\circ}$. Both hemispheres are present in panels.

where $\rho_{0}=m_{p} \cdot 7.3 \cdot 10^{6} \mathrm{~m}^{-3}=1.22 \cdot 10^{-20} \mathrm{kgm}^{-3}$ and $v_{0}=400 \mathrm{~km} / \mathrm{s}$ are chosen as typical solar wind density and velocity; this is for convenience to obtain a correct unit for the power law. $C$ is thus a constant having units of Watts. Because we want to have the power law formula positive and monotonically increasing as a function of negative IMF $B_{z}$, we model the IMF $B_{z}$ inside an exponential. Furthermore, the IMF $B_{z}$ is scaled in the power law by the magnetopause magnetic field given by the pressure balance equation. Table 3 shows the fitted coefficients $C, a, b$, and $d$, together with their error margins and correlation coefficients with the simulation results. Three fits were made: output $P_{\text {ionosphere }}$ taken to be only the Joule heating, only precipitation, and for the sum of Joule heating and precipitation. The first block presents the fitted coefficients, as well as coefficients calculated from the scaling law theory in Sect. 2.3, for Joule heating power in the two events. Comparison of the fitted and theoretical values indicates clearly that the simulated Joule heating power fitted to the solar wind data gives larger values for $a$ and $b$ in both events compared to what

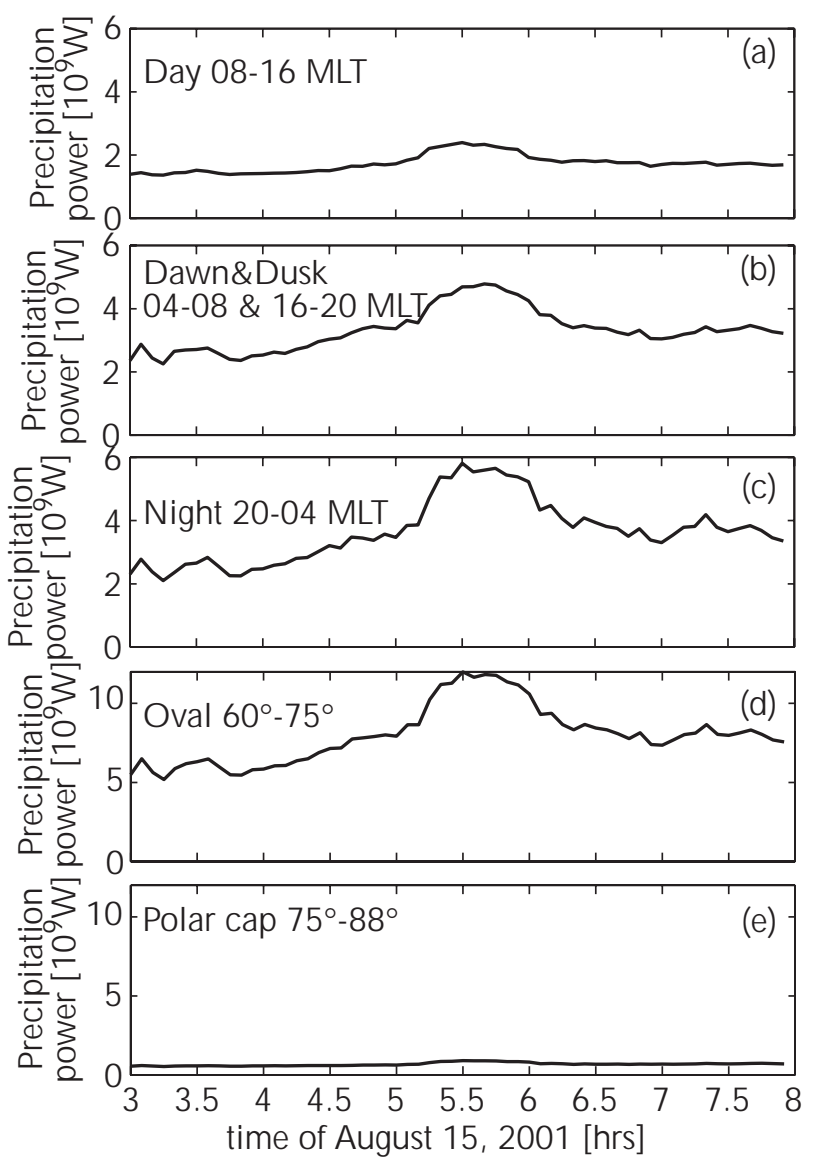

Fig. 12. Precipitation power during the 15 August 2001 substorm in the (a) dayside 08:00-16:00 MLT, (b) dawn 04:00-08:00 MLT and dusk 16:00-20:00 MLT, (c) nightside 20:00-04:00 MLT, (d) oval latitudes $60^{\circ}-75^{\circ}$, and (e) polar cap latitudes $75^{\circ}-88^{\circ}$. Both hemispheres are present in panels.

would be expected from the scaling law theory in Sect. 2.3. In both events, $a$ is roughly the same, but in the April storm simulation $b$ is larger than in the August substorm simulation by a factor of 1.6. This suggests that the solar wind density has roughly the same influence on the deposited Joule heating power, but in the April storm simulation the solar wind speed has much more influence on the deposited Joule heating power than in the August substorm event. However, the coefficient $d$ should be negative, because the Joule heating rate should increase with increasing negative IMF $B_{z}$. As can be seen in Table 3, in the April storm simulation $d$ is positive (but with a large error estimate), and in the August substorm simulation it is negative, indicating that the April storm simulation was more driven by the solar wind dynamic pressure than the IMF direction. Nevertheless, in both events the Joule heating rate calculated using Eq. (10) with the obtained parameters is well-correlated to that given by the simulation; in the April storm event the correlation coefficient is 0.75 , whereas in the August substorm simulation the correlation coefficient is 0.83 . 

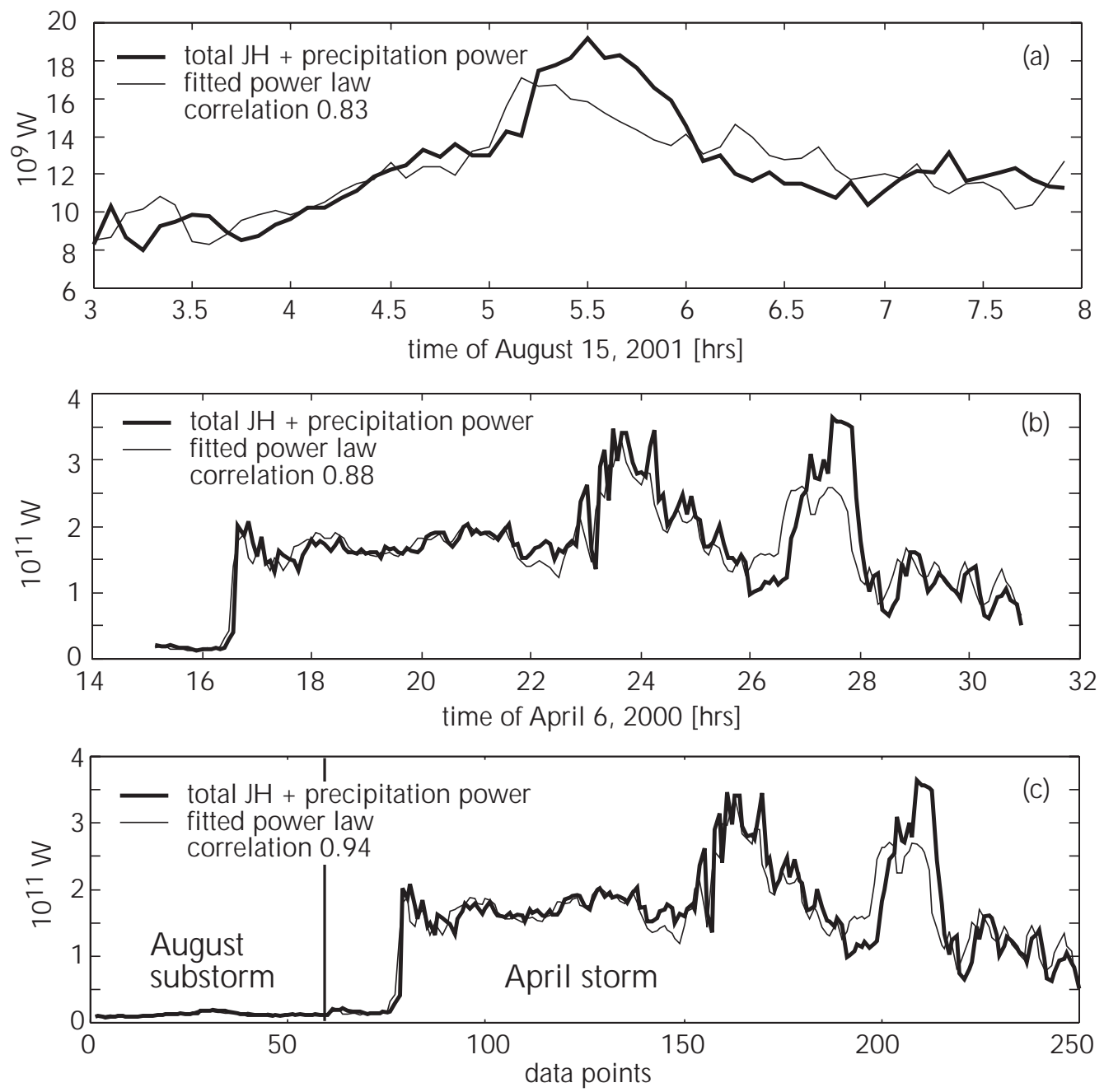

Fig. 13. Sum of Joule heating and precipitation powers (thick line) and fitted curve from Eq. (10) (thin line) for (a) 15 August 2001 substorm simulation, (b) 6 April 2000 storm simulation, and (c) both events merged to the same data vector. Note the different horizontal axis.

The second block of Table 3 presents the results for fitting the solar wind data into the precipitation power calculated in the simulation. The scaling law theory in Sect. 2.3 now predicts a larger $a$ than is obtained by fitting the solar wind data into the precipitation in the simulation. In the August substorm simulation $a$ is comparable in size with $a$ in the April storm simulation. The coefficient $b$ is comparable in size in both events, it is also roughly the same as what the scaling law theory predicts. Curiously, now the coefficient $d$ is negative in the April storm simulation and positive in the $\mathrm{Au}-$ gust substorm simulation, indicating that the IMF direction would have influence on the deposited precipitation power during the April storm, but during the August substorm the deposited precipitation would be almost independent of the IMF direction. Note, however, that the error estimate is large for the positive $d$ coefficient in the substorm case. In both events, the precipitation power calculated using Eq. (10) is well-correlated to what is calculated in the simulation, the correlation coefficient being 0.86 for both events.
We further calculated the sum of Joule heating and precipitation powers, and fitted that sum to the solar wind parameters using Eq. (10). The results are presented in the third block of Table 3. Now, $a, b$, and $d$ are roughly the same for both events, $d$ is negative, the error estimates are small, and the correlation coefficient between the ionospheric dissipation calculated from Eq. (10) and from the simulation is over 0.8. We also merged the two events into one data set, and obtained roughly the same $a, b$, and $d$ with a correlation coefficient 0.94 . All the coefficients are also roughly comparable to the geometric mean of Joule heating and precipitation power scaling exponents. The results are also presented in Fig. 13, where the sum of Joule heating and precipitation power is plotted (thick line) with the ionospheric dissipation calculated from Eq. (10) using the solar wind parameters and coefficients given in Table 3 (thin line) for both events separately (Fig. $13 \mathrm{a}$ and b) and for a data vector where the August substorm data is followed by the data from the April storm (Fig. 13c). Thus, Eq. (10) can describe the total power consumed by the ionosphere much better than its two con- 
tributing pieces (precipitation and Joule heating) separately; also the obtained exponents are closer to the scaling law predictions.

\section{Summary and discussion}

In this paper we have calculated the power dissipated into the ionosphere in a global MHD simulation during two events, a magnetic storm and a magnetospheric substorm. We have studied the latitudinal and longitudinal distribution of the ionospheric dissipation from two parameters, the Joule heating and the particle precipitation. Furthermore, we have compared the results to empirical proxies of the Joule heating and precipitation powers. Finally, we have obtained a power law which predicts the total power deposited into the ionosphere from the solar wind parameters with high correlation between the actual simulation results.

Lu et al. (1998) calculated the Joule heating and precipitation powers during a magnetic storm using the AMIE technique. They obtained a globally integrated average of $190 \mathrm{GW}$ for Joule heating, and about $90 \mathrm{GW}$ for particle precipitation during a 2-day storm period in January 1997. Compared to our results in Fig. 3 and Table 1, the Joule heating in the MHD simulation consumes less than $100 \mathrm{GW}$, with precipitation about $100 \mathrm{GW}$ in both hemispheres during the storm. Therefore, the MHD simulation produces less Joule heating than the AMIE technique, even though the storm modeled in Lu et al. (1998) $\left(D_{s t}\right.$ peak $\left.-85 \mathrm{nT}\right)$ was much smaller than the April 2000 storm. Precipitation in the simulation deposits roughly the same amount of power as that obtained from the AMIE technique. Furthermore, in the $\mathrm{Lu}$ et al. (1998) analysis, the peaks in the Joule heating and the precipitation powers are reached at a time when there is a sudden change in the dynamic pressure during the southward IMF period. In the MHD simulation, the peak in the precipitation power occurred during the main phase and southward IMF, also as a response to a solar wind pressure pulse. The peak in the Joule heating power, however, occurs during the largest dynamic pressure pulse, which took place during the storm recovery phase and northward IMF. A closer examination of the simulation results around 03:00 UT (the time of the maximum Joule heating) reveals that at that time an interhemispherical current system, presumably the result of different conductivities between the two hemispheres, develops in the simulation. Such a current system, is likely to cause a large amount of Joule heat, because in an interhemispherical current system the current closes over a large distance from the one hemisphere to another. This somewhat unexpected result warrants further study.

The Joule heating power calculated from the MHD simulation is clearly different from the empirical proxy by Ahn et al. (1983) in the April 2000 storm event. The temporal variation of the Joule heating power in the MHD simulation resembles remarkably the temporal variation of the solar wind dynamic pressure, whereas the Joule heating power in the Ahn et al. (1983) formula is a scaled $A E$ index. Janhunen and Koski- nen (1997) reported that in the GUMICS-3 MHD simulation (earlier version of GUMICS-4) the Region 1 current system largely closes into the dayside magnetopause currents, which are known to be modified by the solar wind dynamic pressure. Furthermore, the temporal variation of the field-aligned currents in GUMICS-4 (not shown) also resembles the temporal variation of the solar wind dynamic pressure. Therefore, it is natural that the Joule heating power, which depends on the square of the field-aligned currents, also follows the solar wind dynamic pressure variations in the GUMICS-4 MHD simulation. The closure of Region 1 currents to magnetopause currents was also noticed by Siscoe et al. (2002).

The temporal variation of the calculated precipitation power during both events, as well as the temporal variation of Joule heating power during the substorm event, is wellcorrelated with the temporal variation of the empirical proxies of Østgaard et al. (2002) and Ahn et al. (1983). However, the amount of energy deposited into the ionosphere in the simulation is much smaller than the power deposited into the ionosphere in the empirical proxies. The Joule heating power deposited during the storm simulation is $25 \%$ of the Joule heating power given by the Ahn et al. (1983) formula. The precipitation power deposited into the ionosphere is about $50 \%$ of the precipitation power using the Østgaard et al. (2002) formula. The situation is worse in the substorm simulation. The amount of Joule heating power deposited into the ionosphere in the simulation is only $4 \%$ of the amount of Joule heating power according to the Ahn et al. (1983) formula. The amount of precipitation power is about $10 \%$ of the amount of precipitation power according to the Østgaard et al. (2002) formula.

The April 2000 storm was so intense that the oval was located further equatorward than is usual, which means that part of the oval was equatorward of the ionospheric mapping of the inner boundary of the GUMICS-4 MHD simulation $\left(3.7 R_{E}\right)$. Therefore, a major part of nightside precipitation during the storm is not included in the simulation. On the other hand, the empirical proxy used in this study (Østgaard et al., 2002) also gives the precipitation power using the $A L$ index, which is calculated from magnetometer stations located poleward of the main part of the oval. Thus, there are large uncertainties in both the MHD result and in the empirical proxy. In the April 2000 storm case, the amount of precipitation power calculated from the Østgaard et al. (2002) proxy is likely underestimated. The peak value of the precipitation power in the Østgaard et al. (2002) proxy is about $400 \mathrm{GW}$ in the major storm of April 2000, and about $150 \mathrm{GW}$ in a moderate localized substorm. A factor of 2-3 increase from a localized substorm to a major storm with the whole oval filled with auroras appears to be too small. A likely reason for this is that Østgaard et al. (2002) fitted the measured precipitation to the square root of the $A L$ index, which increases slowly for very large values of energy input. For final comparison of the precipitation powers we will simulate an event where the precipitation power is available directly from UVI measurements on board the Polar satellite. 
Table 3. Power law fittings.

\begin{tabular}{lllllll}
\hline & $\mathrm{C}[\mathrm{GW}]$ & $a$ & $b$ & $d$ & \multicolumn{2}{l}{ corr. } \\
\hline Scaling law & & 0.67 & 1.33 & & & $\mathrm{JH}$ \\
April & $4.5 \cdot(1 \pm 0.09)$ & $1.21 \pm 0.08$ & $3.90 \pm 0.33$ & $0.57 \pm 0.38$ & 0.76 & $\mathrm{JH}$ \\
August & $11 \cdot(1 \pm 0.24)$ & $1.17 \pm 0.21$ & $2.32 \pm 0.67$ & $-6.05 \pm 0.77$ & 0.83 & $\mathrm{JH}$ \\
\hline Scaling law & & 1.17 & 3.33 & & & Prec. \\
April & $20 \cdot(1 \pm 0.06)$ & $0.36 \pm 0.05$ & $3.03 \pm 0.22$ & $-3.31 \pm 0.25$ & 0.86 & Prec. \\
August & $15 \cdot(1 \pm 0.15)$ & $0.68 \pm 0.13$ & $3.21 \pm 0.41$ & $0.10 \pm 0.47$ & 0.86 & Prec. \\
\hline Scaling law* & & 0.88 & 2.11 & & & JH+Prec. \\
April & $26 \cdot(1 \pm 0.05)$ & $0.75 \pm 0.04$ & $2.89 \pm 0.18$ & $-1.76 \pm 0.21$ & 0.88 & JH+Prec. \\
August & $25 \cdot(1 \pm 0.17)$ & $0.82 \pm 0.14$ & $3.03 \pm 0.47$ & $-1.75 \pm 0.53$ & 0.83 & JH+Prec. \\
\hline Both events & $25 \cdot(1 \pm 0.03)$ & $0.80 \pm 0.03$ & $2.87 \pm 0.16$ & $-1.81 \pm 0.18$ & 0.94 & JH+Prec. \\
\hline
\end{tabular}

* Geometric mean of Joule heating and precipitation power scaling exponents.

Practically all studies concerning the ionospheric dissipation have reached the conclusion that Joule heating deposits more energy than precipitation (e.g. Lu et al., 1998). Our result shows that in both events the precipitation deposits, on average, slightly more energy than the Joule heating. The underestimation of Joule heating in GUMICS-4 is a consequence of several different sources, one of which can be the typically 20-30\% lower polar cap potentials as compared to, for example, SuperDARN results. As the Joule heating is given by $\Sigma_{P} E^{2}$, underestimation of the polar cap potential by $30 \%$ leads to underestimation in the Joule heating by $\sim 50 \%$. For example, during the April 2000 storm, the average polar cap potential during the main phase according to SuperDARN was about $80 \mathrm{kV}$, whereas the GUMICS-4 result was about $50 \mathrm{kV}$, giving 2.56 for the ratio of polar cap potentials squared. The Joule heating rate during the April 2000 storm main phase multiplied by 2.56 gives $\sim 2 \cdot 10^{11} \mathrm{~W}$, which is now of the same order of magnitude as the Ahn et al. (1983) value (Fig. 3). Similarly, during the 15 August 2001 substorm event, the polar cap potential was steadily about $50 \mathrm{kV}$ (McPherron et al., 2002), whereas GUMICS yielded about $30 \mathrm{kV}$. Again, the ratio of polar cap potentials squared gives 2.8 , and Joule heating multiplied by this value during the substorm is about $1.5 \cdot 10^{10} \mathrm{~W}$, which is still less than that given by Ahn et al. (1983), but of the same order of magnitude.

An additional effect causing the low Joule heating values could be caused by a more localized field-aligned current closure. As a simplified thought experiment, let us consider a single current loop that closes through the ionosphere, i.e. a loop where the acceleration region in the magnetosphere and the ionospheric load are connected in a series. The power consumed in a current loop is determined by the potential difference and the total current $(P=U I)$. In a single current loop the same current flows through the acceleration region and the ionospheric load, and, therefore, $P_{\text {accel }} / U_{\text {accel }}=$ $P_{\text {ionosph }} / U_{\text {ionosph }}$. The characteristic energy of precipitating electrons determines that $P_{\text {accel }}=P_{\text {prec }}$ and $P_{\text {ionosph }}=P_{J H}$, we obtain $P_{\text {prec }} / P_{J H}=U_{\text {accel }} / U_{\text {iono }}$. In both simulated cases
$P_{\text {prec }} / P_{J H}>1$, which means that $U_{\text {accel }} / U_{\text {iono }}>1$, indicating that the potential difference in the ionosphere is smaller than in the acceleration region, suggesting that the current closes over a relatively short distance in the ionosphere. Therefore, the fact that Joule heating is a smaller energy sink than precipitation in the global MHD simulation might indicate that in the simulation the currents could close over a short distance in the ionosphere. Satellite observations reported by Marklund et al. (1998) demonstrate that the closure of a part of the field-aligned currents in the substorm current wedge occurs locally near the surge head. Furthermore, a small error in the GUMICS-4 total Joule heating result can also be caused by the limited ionospheric grid resolution. For example, discrete intensive arcs with sizes below the GUMICS-4 ionospheric grid resolution produce locally high values of Joule heating. We estimate roughly that the discrete arcs can increase the total Joule heating up to $10 \%$.

When comparing the latitudinal and longitudinal distributions of the ionospheric dissipation in the two simulated events the differences between the storm and substorm cases become evident. In short, the dayside has a dominant role in the storm case, whereas the nightside oval dissipates most of the power in the substorm case. Another clear distinction can be seen in the Tables 1 and 2. While in the storm case the Joule heating and the precipitation dissipate roughly the same amount of energy into the ionosphere, during the substorm precipitation energy exceeds that of Joule heating. This can partly be explained by the fact that a large part of the precipitation is not included in the storm simulation, due to the 3.7 $R_{E}$ inner boundary of the magnetospheric domain, which leaves part of the oval equatorward of that boundary.

The final topic of this paper, the development of a simple power law between the solar wind input and the ionospheric output, proved to be a fruitful starting point in using global MHD simulations in investigating the energy transfer and dissipation. We found a remarkably high correlation coefficient for the power law that calculates the total ionospheric dissipation from the solar wind input, using the same exponents for the two events. The power law works best for the 
sum of Joule heating and precipitation power, which is natural as the two parameters are not independent of each other. The relative importance of the exponents $a, b$, and $d$ suggests that the solar wind density and velocity have more impact on the total ionospheric dissipation than the IMF $B_{z}$. This may not be universally true. Coincidentally, in the two chosen events the impact of the solar wind pressure was stronger than the IMF. In the April 2000 storm the solar wind dynamic pressure was unusually high, and in the moderate August 2001 substorm the IMF $B_{z}$ was quite weak and not rapidly varying. This suggests that more events with different inputs must be simulated, to obtain a power law that would recover the earlier empirically strong correlation found between energy dissipation and IMF $B_{z}$.

Acknowledgements. We thank the NSSDC for maintaining the CDAWeb facility through which Geotail data for the simulation input were obtained. The CANOPUS instrument array was constructed and is maintained and operated by the Canadian Space Agency for the Canadian scientific community. The work of M. P. and P. J. is supported by the MaDaMe program of the Academy of Finland.

The Editor in Chief thanks M. Wiltberger and another referee for their help in evaluating this paper.

\section{References}

Akasofu, S.-I.: Energy coupling between the solar wind and the magnetosphere, Space Sci. Rev., 28, 121-190, 1981.

Acuña, M. H., Ogilivie, K. W., Baker, D. N., Curtis, S. A., Fairfield, D. H., and Mish, W. H.: The global geospace science program and its investigations, Space Sci. Rev., 71, 5-21, 1995.

Ahn, B.-H. J., Akasofu, S. -I., and Kamide, Y.: The Joule heat production rate and the particle energy injection rate as function of the geomagnetic indices $A E$ and $A L$, J. Geophys. Res., 88, 6275-6287, 1983.

Axford, W. I. and Hines, C. O.: A unifying theory of high-latitude geophysical phenomena and geomagnetic storms, Can. J. Phys., 39, 1433, 1961.

Dungey, J. W.: Interplanetary magnetic field and the auroral zones, Phys. Rev. Lett., 6, 47-48, 1961.

Huttunen, K. E. J., Koskinen, H. E. J., Pulkkinen, T. I., Pulkkinen, A., Palmroth, M., Reeves, G. D., and Singer, H. J.: April 2000 magnetic storm: Solar wind driver and magnetospheric response, J. Geophys. Res., 107, doi:10.1029/2001JA009154, 2002.

Janhunen, P.: GUMICS-3-A global ionosphere-magnetosphere coupling simulation with high ionospheric resolution, in: Proc. Environmental Modelling for Space-Based Applications, Sept. 18-20 1996 (ESTEC, The Netherlands, ESA SP-392,) 1996.

Janhunen, P. and Koskinen, H. E. J.: The closure of Region-1 field-aligned current in MHD simulation, Geophys. Re. Lett., 24, 1419-1422, 1997.

Janhunen, P. and Olsson, A.: The current-voltage relationship revisited: exact and approximate formulas with almost general validity for hot magnetospheric electrons for bi-Maxwellian and kappa distributions, Ann. Geophysicae, 16, 292-297, 1998.

Lu, G., Baker, D N., McPherron, R. L., Farrugia, C. J, Lummerzheim, D., Ruohoniemi, J. M., Rich, F. J., Evans, D. S., Lepping, R. P., Brittnacher, M., Li, X., Greenwald, R., Sofko, G., Villain, J., Lester, M., Thayer, J., Moretto, T., Milling, D.,
Troshichev, O., Zaitzev, A., Odintzov, V., Makarov, G., and Hayashi, K.: Global energy deposition during the January 1997 magnetic cloud event, J. Geophys. Res., 103, 11 685-11 694, 1998.

Marklund, G. T., Karlsson, T., Blomberg, L. G., Lindqvist, P.-A., Fälthammar, C.-G., Johnson, M. L., Murphree, J. S., Andersson, L., Eliasson, L., Opgenoorth, H. J., and Zanetti, L. J.: Observations of the electric field fine structure associated with the westward traveling surge and large-scale auroral spirals, J. Geophys. Res., 103, 4125-4144, 1998.

McPherron, R. L., Kivelson, M. G., Khurana, K., Amm, O., Baker, J. B., Balogh, A., Rème, H., Connors, M., Creutzberg, F., Dandouras, I., Mann, I., Milling, D., Moldwin, M. B., Rostoker, G., Russell, C. T., and Singer, H.: Cluster observations of the postmidnight plasma sheet at 18 Re during substorms, in: Proceedings of the Sixth International Conference on Substorms, edited by Winglee, R. M., University of Washington, Seattle, USA, March 25-29, 283-290, 2002.

Newell, P. T., Lyons, K. M., and Meng, C.-I.: A large survey of electron acceleration events, J. Geophys. Res., 101, 2599-2614, 1996.

Østgaard, N. R., Vondrak, R., Gjerloev, J. W., and Germany, G. A.: A relation between the energy deposition by electron precipitation and geomagnetic indices during substorms, J. Geophys. Res. 107(A9), 1246, doi: 10.1029/2001JA002003, $2002 .$.

Palmroth, M., Janhunen, P., Pulkkinen, T. I., and Peterson, W. K.: Cusp and magnetopause locations in global MHD simulation, J. Geophys. Res., 106, 29 435-29 450, 2001.

Palmroth, M., Pulkkinen, T. I., Janhunen, P., and Wu, C. C.: Stormtime energy transfer in global MHD simulation, J. Geophys. Res., 108(A1), 1048, doi:10.1029/2002JA009446, 2003.

Papadopoulos, K., Goodrich, C., Wiltberger, M., Lopez, R., and Lyon, J. G.: The physics of substorms as revealed by the ISTP, Phys. Chem. Earth (c), 24, 189-202, 1999.

Pulkkinen, T. I., Ganushkina, N. Yu., Tanskanen, E. I., Lu, G., Baker, D. N., Turner, N. E., Fritz, T. A., Fennell, J. F., and Roeder, J.: Energy dissipation during a geomagnetic storm: May 1998, Adv. Space Res., 30, 2231-2240, 2002.

Robinson, R. M., Vondrak, R. R., Miller, K., Dabbs, T., and Hardy, D.: On calculating ionospheric conductances from he flux and energy of precipitating electrons, J. Geophys. Res., 92, 25652569, 1987.

Richmond, A. D. and Kamide, Y.: Mapping electrodynamic features of the high-latitude ionosphere from localized observationsTechnique, J. Geophys. Res., 93, 5741-5759, 1988.

Scurry, L. and Russell, C. T.: Proxy studies of energy trasnfer to the magnetosphere, J. Geophys. Res., 96, 9541-9548, 1991.

Siscoe, G. L., Crooker, N. U., and Siebert, K. D.: Transpolar potential saturation: Roles of Region 1 current system and solar wind ram pressure, J. Geophys. Res., 107(A10), 1321, doi:10.1029/2001JA009176, 2002.

Turner, N. E., Baker, D. N., Pulkkinen, T. I., Roeder, J. L., Fennell, J. F., and Jordanova, V. K.: Energy content in the storm time ring current, J. Geophys. Res., 106, 19 149-19 156, 2001.

Walker, R. J., Ogino, T., Raeder, J., and Ashour-Abdalla, M.: A global magnetohydrodynamic simulation of the magnetosphere when the interplanetary magnetic field is southward: The onset of magnetotail reconnection, J. Geophys. Res., 98, 17235 $17249,1993$.

Weiss, L. A., Reiff, P. H., Moses, J. J., Heelis, R. A., and Moore, B. D.: Energy dissipation in substorms, in: Proc. of International Conference on Substorms 1, ESA SP-335, 1992. 